Supporting Information for

\title{
Asymmetric Reduction of $\beta$-Ketonitriles with a Recombinant Carbonyl Reductase and Cascade Enzymatic Transformation to Optically Pure $\beta$-Hydroxy Carboxylic Acids
}

Dunming Zhu, Haribabu Ankati, Chandrani Mukherjee, Yan Yang, Edward R. Biehl and Ling Hua

Department of Chemistry, Southern Methodist University, Dallas, Texas 75275, USA

\section{Table of contents}

1. General methods $\quad$ S2

2. Carbonyl reductase-catalyzed reduction of $\beta$-ketonitriles $\quad$ S2

3. Nitrilase-catalyzed hydrolysis of $\beta$-hydroxy nitriles $\quad$ S2

4. NMR and specific rotation data for $\beta$-hydroxy nitriles

$\begin{array}{ll}\text { and } \beta \text {-hydroxy carboxylic acids } & \text { S2-S5 }\end{array}$

5. References $\quad$ S5

6. ${ }^{13} \mathrm{C}$ NMR spectra of $\beta$-hydroxy nitriles and $\beta$-hydroxy carboxylic acids $\quad$ S6-S26 
1. General methods. The chiral HPLC analysis was performed on an Agilent 1100 series high-performance liquid chromatography system with (S,S)-Whelk-O 1 column $(25 \mathrm{~cm} \times 4.6 \mathrm{~mm}$, Regis Technologies Inc.). The chiral GC analysis was performed on a Hewlett Packard 5890 series II plus gas chromatograph equipped with autosampler, EPC, split/splitless injector, FID detector and CP-Chirasil-Dex CB chiral capillary column (25 $\mathrm{m} \times 0.25 \mathrm{~mm})$. Aromatic $\beta$-ketonitriles were prepared and purified as previously reported. ${ }^{1}$ The carbonyl reductase from Candida magnoliae $(\mathbf{C M C R}),{ }^{2}$ nitrilases from cyanobacterium Synechocystis sp. strain PCC 6803 (NIT6803) $^{3}$ and Bradyrhizobium japonicum strain USDA110 (bll6402) ${ }^{4}$ were readily prepared at gram scale using our previously reported procedures.

2. Carbonyl reductase-catalyzed reduction of $\boldsymbol{\beta}$-ketonitriles. The reaction was carried out as follows: D-glucose (400 mg), D-glucose dehydrogenase (10 mg), NADPH $(10 \mathrm{mg})$ and carbonyl reductase $(10 \mathrm{mg})$ were mixed in a potassium phosphate buffer (100 ml, $100 \mathrm{mM}, \mathrm{pH}$ 7.0). To the mixture was added a 3-oxo-3-phenylpropanenitrile (1a) solution (170 mg 1.17 mmole) in $5.0 \mathrm{ml}$ DMSO. The mixture was stirred at room temperature and monitored by TLC until conversion was complete. The mixture was extracted with methyl tert-butyl ether. The organic extract was dried over anhydrous sodium sulfate and removal of the solvent gave (R)-3-hydroxy-3-phenylpropanenitrile (2a, $147 \mathrm{mg}, 85 \%$ yield), which was identified by comparison of ${ }^{1} \mathrm{H}$ and ${ }^{13} \mathrm{C}$ NMR with literature data. ${ }^{1,5-7}$ The absolute configurations of the product alcohols were determined by comparison of the sign of specific rotation data with those in the literature. ${ }^{1,8}$

3. Nitrilase-catalyzed hydrolysis of $\boldsymbol{\beta}$-hydroxy nitriles. The reaction was carried out as follows: (R)-3-Hydroxy-3-phenylpropanenitrile (2a, $70 \mathrm{mg}, 0.34 \mathrm{mmole}$ ) was suspended in $50 \mathrm{ml}$ of potassium phosphate buffer $(100 \mathrm{mM}, \mathrm{pH}$ 7.2). Nitrilase NIT6803 $\left(3 \mathrm{mg}\right.$ ) was added to the mixture, which was stirred at $30^{\circ} \mathrm{C}$. The reaction was monitored by TLC. After completion of the reaction, the reaction mixture was acidified with $2 \mathrm{~N}$ $\mathrm{HCl}$, saturated with $\mathrm{NaCl}$, and extracted with ethylacetate. The organic extract was dried over sodium sulfate and evaporated under reduced pressure to give crude product, which was purified by preparative TLC. The product (R)-3-hydroxy-3-phenylpropanoic acid (3a) was obtained as white crystalline solid (67 $\mathrm{mg}, 85 \%$ yield), which was identified by comparison of ${ }^{1} \mathrm{H}$ and ${ }^{13} \mathrm{C}$ NMR with literature data. ${ }^{1,9,10}$ The absolute configurations of the product alcohols were determined by comparison of the sign of specific rotation data with those in the literature. ${ }^{1,11}$

\section{NMR and specific rotation data for $\beta$-hydroxy nitriles and $\beta$-hydroxy carboxylic acids:}

(R)-3-Hydroxy-3-phenylpropanenitrile (2a). ${ }^{1}$ viscous oil, $147 \mathrm{mg}, 85 \%$ yield; ${ }^{1} \mathrm{H}$ NMR $\left(\mathrm{CDCl}_{3}\right) \delta(\mathrm{ppm}) 2.66\left(\mathrm{~d}, J=6.0 \mathrm{~Hz}, 2 \mathrm{H}, \mathrm{CH}_{2}\right), 3.45(\mathrm{br}, 1 \mathrm{H}, \mathrm{OH}), 4.93(\mathrm{t}, J=8.0 \mathrm{~Hz}$, $1 \mathrm{H}, \mathrm{CH}), 7.29-7.37\left(\mathrm{~m}, 5 \mathrm{H}\right.$, aromatic); ${ }^{13} \mathrm{C}$ NMR $\left(\mathrm{CDCl}_{3}\right) \delta(\mathrm{ppm}) 27.7\left(\mathrm{CH}_{2}\right), 69.5$ $(\mathrm{CH}), 117.5(\mathrm{CN}), 125.4(\mathrm{CH}), 128.5(\mathrm{CH}), 128.7(\mathrm{CH}), 140.9(\mathrm{C}) ;[\alpha]^{22}{ }_{\mathrm{D}}+44.7(\mathrm{c} 2.50$, $\left.\mathrm{CH}_{3} \mathrm{OH}\right) ;$ lit. $^{11}[\alpha]^{22}{ }_{\mathrm{D}}+50.4\left(\mathrm{c} 4.945, \mathrm{CH}_{3} \mathrm{OH}\right)$.

$(\boldsymbol{R})$-3-Hydroxy-3-phenylpropanoic acid (3a). ${ }^{1}$ white crystalline solid, $67 \mathrm{mg}, 85 \%$ yield; m.p. $117-120^{\circ} \mathrm{C} .{ }^{1} \mathrm{H}$ NMR (methanol- $\left.\mathrm{d}_{4}\right) \delta(\mathrm{ppm})$ 2.62-2.66 (m, $2 \mathrm{H}, \mathrm{CH}_{2}$ ), 5.04- 
$5.07(\mathrm{~m}, 1 \mathrm{H}, \mathrm{CH}), 7.20-7.25\left(\mathrm{~m}, 5 \mathrm{H}\right.$, aromatic); ${ }^{13} \mathrm{C}$ NMR (methanol- $\left.\mathrm{d}_{4}\right) \delta(\mathrm{ppm}) 42.3$ $\left(\mathrm{CH}_{2}\right), 69.1(\mathrm{CH}), 124.4(\mathrm{CH}), 126.0(\mathrm{C}), 126.8(\mathrm{CH}), 142.6(\mathrm{C}), 172.4(\mathrm{CO}) ;[\alpha]^{22}{ }_{\mathrm{D}}$ $+23.8\left(\mathrm{c} 0.5, \mathrm{CH}_{3} \mathrm{OH}\right) ;$ lit. $^{11}[\alpha]^{22}{ }_{\mathrm{D}}+22.4\left(\mathrm{c} 4.135, \mathrm{CH}_{3} \mathrm{OH}\right)$.

(R)-3-(4'-Fluorophenyl)-3-hydroxypropanenitrile $(\mathbf{2 b}) .{ }^{1}$ colorless oil, $155 \mathrm{mg}, 90 \%$ yield; ${ }^{1} \mathrm{H}$ NMR $\left(\mathrm{CDCl}_{3}\right) \delta(\mathrm{ppm}) 2.75\left(\mathrm{~d}, J=6.2 \mathrm{~Hz}, 2 \mathrm{H}, \mathrm{CH}_{2}\right), 2.85$ (brs, $\left.1 \mathrm{H}, \mathrm{OH}\right), 5.02$ $5.05(\mathrm{~m}, 1 \mathrm{H}, \mathrm{CH}), 7.10(\mathrm{~d}, J=8.6 \mathrm{~Hz}, 2 \mathrm{H}$, aromatic), $7.38(\mathrm{~d}, J=8.6 \mathrm{~Hz}, 2 \mathrm{H}$, aromatic); ${ }^{13} \mathrm{C} \mathrm{NMR}\left(\mathrm{CDCl}_{3}\right) \delta(\mathrm{ppm}) 28.4\left(\mathrm{CH}_{2}\right), 69.7(\mathrm{CH}), 116.2\left(\mathrm{~d},{ }^{2} J_{\mathrm{C}-\mathrm{F}}=21 \mathrm{~Hz}, \mathrm{CH}\right), 117.6$ $(\mathrm{CN}), 127.8\left(\mathrm{~d},{ }^{3} J_{\mathrm{C}-\mathrm{F}}=8 \mathrm{~Hz}, \mathrm{CH}\right), 137.2(\mathrm{C}), 163.2\left(\mathrm{~d},{ }^{1} J_{\mathrm{C}-\mathrm{F}}=246 \mathrm{~Hz}, \mathrm{C}\right) ;[\alpha]^{22}{ }_{\mathrm{D}}+38.43$ (c $\left.0.75, \mathrm{CH}_{3} \mathrm{OH}\right)$.

(R)-3-(4'-Fluorophenyl)-3-hydroxypropanoic acid (3b). ${ }^{1}$ white solid, $48 \mathrm{mg}, 86 \%$ yield; m.p. $70-72{ }^{\circ} \mathrm{C} .{ }^{1} \mathrm{H}$ NMR $\left(\mathrm{CDCl}_{3}\right) \delta(\mathrm{ppm})$ 2.72-2.86 (m, $\left.2 \mathrm{H}, \mathrm{CH}_{2}\right), 5.13-5.16(\mathrm{~m}$, $1 \mathrm{H}, \mathrm{CH}), 7.03-7.07\left(\mathrm{~m}, 2 \mathrm{H}\right.$, aromatic), 7.34-7.38 (m, $2 \mathrm{H}$, aromatic); ${ }^{13} \mathrm{C} \mathrm{NMR}\left(\mathrm{CDCl}_{3}\right) \delta$ (ppm) $42.9\left(\mathrm{CH}_{2}\right), 69.76(\mathrm{CH}), 115.6\left(\mathrm{~d},{ }^{2} J_{\mathrm{C}-\mathrm{F}}=21 \mathrm{~Hz}, \mathrm{CH}\right), 127.4\left(\mathrm{~d},{ }^{3} J_{\mathrm{C}-\mathrm{F}}=8 \mathrm{~Hz}, \mathrm{CH}\right)$, $137.8(\mathrm{C}), 163.2\left(\mathrm{~d},{ }^{1} J_{\mathrm{C}-\mathrm{F}}=245 \mathrm{~Hz}, \mathrm{C}\right), 176.5(\mathrm{CO}) ;[\alpha]^{22}{ }_{\mathrm{D}}+9.6\left(\mathrm{c} 0.33, \mathrm{CH}_{3} \mathrm{OH}\right)$.

(R)-3-(2',4'-Difluorophenyl)-3-hydroxypropanenitrile (2c) ${ }^{5}$ colorless oil, $158 \mathrm{mg}$, 92\% yield; ${ }^{1} \mathrm{H}$ NMR $\left(\mathrm{CDCl}_{3}\right) \delta(\mathrm{ppm}) 2.50$ (brs, $\left.1 \mathrm{H}, \mathrm{OH}\right), 2.66-2.82\left(\mathrm{~m}, 2 \mathrm{H}, \mathrm{CH}_{2}\right), 5.26$ (br, $1 \mathrm{H}, \mathrm{CH}), 6.73-6.90\left(\mathrm{~m}, 2 \mathrm{H}\right.$, aromatic), 7.49-7.53 (m, 1H, aromatic); ${ }^{13} \mathrm{C}$ NMR $\left(\mathrm{CDCl}_{3}\right) \delta(\mathrm{ppm}) 26.9\left(\mathrm{CH}_{2}\right), 63.8(\mathrm{CH}), 104.1\left(\mathrm{t},{ }^{2} J_{\mathrm{C}-\mathrm{F}}=25 \mathrm{~Hz}, \mathrm{CH}\right), 112.0\left(\mathrm{dd},{ }^{2} J_{\mathrm{C}-\mathrm{F}}=\right.$ $\left.21 \mathrm{~Hz},{ }^{3} J_{\mathrm{C}-\mathrm{F}}=3 \mathrm{~Hz}, \mathrm{CH}\right), 116.7(\mathrm{CN}), 123.9\left(\mathrm{dd},{ }^{2} J_{\mathrm{C}-\mathrm{F}}=13 \mathrm{~Hz},{ }^{3} J_{\mathrm{C}-\mathrm{F}}=4 \mathrm{~Hz}, \mathrm{C}\right), 128.2$ $\left(\mathrm{dd},{ }^{3} J_{\mathrm{C}-\mathrm{F}}=10 \mathrm{~Hz},{ }^{3} J_{\mathrm{C}-\mathrm{F}}=5 \mathrm{~Hz}, \mathrm{CH}\right), 159.2\left(\mathrm{dd},{ }^{1} J_{\mathrm{C}-\mathrm{F}}=249 \mathrm{~Hz},{ }^{3} J_{\mathrm{C}-\mathrm{F}}=12 \mathrm{~Hz}, \mathrm{C}\right), 163.2$ (dd, $\left.{ }^{1} J_{\mathrm{C}-\mathrm{F}}=249 \mathrm{~Hz},{ }^{3} J_{\mathrm{C}-\mathrm{F}}=12 \mathrm{~Hz}, \mathrm{C}\right) ;[\alpha]^{22}{ }_{\mathrm{D}}+46.85\left(\mathrm{c} 1.67, \mathrm{CH}_{3} \mathrm{OH}\right)$.

(R)-3-(2',4'-Difluorophenyl)-3-hydroxypropanoic acid (3c). ${ }^{9}$ viscous oil, $89 \mathrm{mg}, 89 \%$ yield; ${ }^{1} \mathrm{H}$ NMR (methanol- $\left.\mathrm{d}_{4}\right) \delta(\mathrm{ppm}) 2.62-2.64\left(\mathrm{~m}, 2 \mathrm{H}, \mathrm{CH}_{2}\right), 5.28-5.32(\mathrm{~m}, 1 \mathrm{H}, \mathrm{CH})$, 6.82-6.92 (m, $2 \mathrm{H}$, aromatic), 7.46-7.50 (m, $1 \mathrm{H}$, aromatic); ${ }^{13} \mathrm{C}$ NMR (methanol- $\left.\mathrm{d}_{4}\right) \delta$ (ppm) $41.0\left(\mathrm{CH}_{2}\right), 62.6(\mathrm{CH}), 101.8\left(\mathrm{t},{ }^{2} J_{\mathrm{C}-\mathrm{F}}=25 \mathrm{~Hz}, \mathrm{CH}\right), 109.7\left(\mathrm{~d},{ }^{2} J_{\mathrm{C}-\mathrm{F}}=21 \mathrm{~Hz}, \mathrm{CH}\right)$, $125.8\left(\mathrm{~d},{ }^{2} J_{\mathrm{C}-\mathrm{F}}=10 \mathrm{~Hz}, \mathrm{C}\right), 127.3\left(\mathrm{t},{ }^{3} J_{\mathrm{C}-\mathrm{F}}=9 \mathrm{~Hz}, \mathrm{CH}\right), 159.7\left(\mathrm{~d},{ }^{1} J_{\mathrm{C}-\mathrm{F}}=257 \mathrm{~Hz}, \mathrm{C}\right)$, $162.4\left(\mathrm{~d},{ }^{1} J_{\mathrm{C}-\mathrm{F}}=259 \mathrm{~Hz}, \mathrm{C}\right), 171.9(\mathrm{CO}) ;[\alpha]^{22}{ }_{\mathrm{D}}+34.6\left(\mathrm{c} 0.66, \mathrm{CH}_{3} \mathrm{OH}\right)$.

(R)-3-(4'-Chlorophenyl)-3-hydroxypropanenitrile (2d). ${ }^{1}$ viscous oil, $155 \mathrm{mg}, 90 \%$ yield; ${ }^{1} \mathrm{H}$ NMR $\left(\mathrm{CDCl}_{3}\right) \delta(\mathrm{ppm})$ 2.63-2.65 (m, $\left.2 \mathrm{H}, \mathrm{CH}_{2}\right), 3.0$ (brs, $\left.1 \mathrm{H}, \mathrm{OH}\right), 4.91$ (br, $1 \mathrm{H}, \mathrm{CH}), 7.18-7.29\left(\mathrm{~m}, 4 \mathrm{H}\right.$, aromatic); ${ }^{13} \mathrm{C}$ NMR $\left(\mathrm{CDCl}_{3}\right) \delta(\mathrm{ppm}) 27.8\left(\mathrm{CH}_{2}\right), 69.1$ $(\mathrm{CH}), 117.1(\mathrm{CN}), 126.9(\mathrm{CH}), 128.9(\mathrm{CH}), 134.4(\mathrm{C}), 139.4(\mathrm{C}) ;[\alpha]^{22}{ }_{\mathrm{D}}+53.20(\mathrm{c} 2.0$, $\mathrm{CH}_{3} \mathrm{OH}$ ); lit. ${ }^{12}[\alpha]^{30}{ }_{\mathrm{D}}-54.5$ (c 2.0, $\mathrm{CHCl}_{3}$ ) for (S)-enantiomer.

(R)-3-(4'-Chlorophenyl)-3-hydroxypropanoic acid (3d). ${ }^{1}$ white chrystalline solid, 67 mg, $86 \%$ yield; m.p. $80-81^{\circ} \mathrm{C} .{ }^{1} \mathrm{H}$ NMR $\left(\mathrm{CDCl}_{3}\right) \delta(\mathrm{ppm}) 2.74-2.86\left(\mathrm{~m}, 2 \mathrm{H}, \mathrm{CH}_{2}\right)$, 5.14$5.17(\mathrm{~m}, 1 \mathrm{H}, \mathrm{CH}), 7.28-7.38\left(\mathrm{~m}, 4 \mathrm{H}\right.$, aromatic); ${ }^{13} \mathrm{C}$ NMR (methanol- $\left.\mathrm{d}_{4}\right) \delta(\mathrm{ppm}) 45.0$ $\left(\mathrm{CH}_{2}\right), 71.1(\mathrm{CH}), 128.7(\mathrm{CH}), 129.6(\mathrm{CH}), 134.3(\mathrm{C}), 142.2(\mathrm{C}), 174.8(\mathrm{CO}) ;[\alpha]^{22}{ }_{\mathrm{D}}$ $+16.7\left(\mathrm{c} 0.5, \mathrm{CH}_{3} \mathrm{OH}\right)$.

(R)-3-(4'-Bromophenyl)-3-hydroxypropanenitrile (2e). ${ }^{5}$ viscous oil, $151 \mathrm{mg}, 88 \%$ yield; ${ }^{1} \mathrm{H}$ NMR $\left(\mathrm{CDCl}_{3}\right) \delta(\mathrm{ppm})$ 2.69-2.75 (m, $\left.2 \mathrm{H}, \mathrm{CH}_{2}\right), 3.33$ (brs, $\left.1 \mathrm{H}, \mathrm{OH}\right), 4.96$ (br, $1 \mathrm{H}, \mathrm{CH}), 7.25(\mathrm{dd}, J=2.0,2.2 \mathrm{~Hz}, 2 \mathrm{H}$, aromatic), $7.50(\mathrm{dd}, J=4.7,2.3 \mathrm{~Hz}, 2 \mathrm{H}$, aromatic); ${ }^{13} \mathrm{C}$ NMR $\left(\mathrm{CDCl}_{3}\right) \delta(\mathrm{ppm}) 28.3\left(\mathrm{CH}_{2}\right), 69.6(\mathrm{CH}), 117.6(\mathrm{CN}), 123.0(\mathrm{C})$, $127.7(\mathrm{CH}), 132.3(\mathrm{CH}), 140.4(\mathrm{C}) ;[\alpha]^{22}+41.90\left(\mathrm{c} 1.0, \mathrm{CH}_{3} \mathrm{OH}\right)$.

(R)-3-(4'-Bromophenyl)-3-hydroxypropanoic acid (3e). ${ }^{9}$ white solid, $77 \mathrm{mg}, 85 \%$ yield; m.p. $110-112^{\circ} \mathrm{C} .{ }^{1} \mathrm{H}$ NMR (methanol-d $\left.\mathrm{d}_{4}\right) \delta(\mathrm{ppm})$ 2.63-2.68 (m, $2 \mathrm{H}, \mathrm{CH}_{2}$ ), 5.04$5.06(\mathrm{~m}, 1 \mathrm{H}, \mathrm{CH}), 7.32(\mathrm{~d}, J=7.9 \mathrm{~Hz}, 2 \mathrm{H}$, aromatic), $7.50(\mathrm{~d}, J=7.9 \mathrm{~Hz}, 2 \mathrm{H}$, aromatic); 
${ }^{13} \mathrm{C}$ NMR (methanol-d $\left.\mathrm{d}_{4}\right) \quad \delta(\mathrm{ppm}) 42.3\left(\mathrm{CH}_{2}\right), 68.5(\mathrm{CH}), 119.6(\mathrm{CH}), 126.4(\mathrm{CH}), 129.9$ $(\mathrm{CH}), 142.1(\mathrm{C}), 172.1(\mathrm{CO}) ;[\alpha]_{\mathrm{D}}^{22}+7.5\left(\mathrm{c} 0.5, \mathrm{CH}_{3} \mathrm{OH}\right)$.

(R)-3-Hydroxy-3-(4'-methylphenyl)propanenitrile (2f). ${ }^{1}$ colorless viscous oil,; 155 mg, 90\% yield; ${ }^{1} \mathrm{H}$ NMR $\left(\mathrm{CDCl}_{3}\right) \delta(\mathrm{ppm}) 2.37\left(\mathrm{~s}, 3 \mathrm{H}, \mathrm{CH}_{3}\right), 2.58-3.01\left(\mathrm{~m}, 2 \mathrm{H}, \mathrm{CH}_{2}\right)$, $2.97(\mathrm{~s}, 1 \mathrm{H}, \mathrm{OH}), 5.0(\mathrm{br}, 1 \mathrm{H}, \mathrm{CH}), 7.20(\mathrm{~d}, J=7.9 \mathrm{~Hz}, 2 \mathrm{H}$, aromatic), $7.25(\mathrm{~d}, J=7.9$ $\mathrm{Hz}, 2 \mathrm{H}$, aromatic); ${ }^{13} \mathrm{C} \mathrm{NMR}\left(\mathrm{CDCl}_{3}\right) \delta(\mathrm{ppm}) 21.0\left(\mathrm{CH}_{3}\right), 27.7\left(\mathrm{CH}_{2}\right), 69.7(\mathrm{CH}), 117.4$ $(\mathrm{CN}), 125.3(\mathrm{CH}), 129.4(\mathrm{CH}), 138.0(\mathrm{C}), 138.4(\mathrm{C}) ;[\alpha]_{\mathrm{D}}^{22}+48.4\left(\mathrm{c} 1.67, \mathrm{CH}_{3} \mathrm{OH}\right)$; lit. ${ }^{12}$ $[\alpha]^{30}-52.6\left(\mathrm{c} 2.0, \mathrm{CHCl}_{3}\right)$ for (S)-enantiomer.

(R)-3-Hydroxy-3-(4'-methylphenyl)propanoic acid (3f). ${ }^{1}$ white solid, $66 \mathrm{mg}, 85 \%$ yield; m.p. 88-91 ${ }^{\circ} \mathrm{C} .{ }^{1} \mathrm{H}$ NMR (methanol- $\left.\mathrm{d}_{4}\right) \delta(\mathrm{ppm}) 2.33\left(\mathrm{~s}, 3 \mathrm{H}, \mathrm{CH}_{3}\right), 2.55-2.68(\mathrm{~m}$, $\left.2 \mathrm{H}, \mathrm{CH}_{2}\right), 4.98-5.01(\mathrm{~m}, 1 \mathrm{H}, \mathrm{CH}), 7.10(\mathrm{~d}, J=7.6 \mathrm{~Hz}, 2 \mathrm{H}$, aromatic), $7.22(\mathrm{~d}, J=7.9 \mathrm{~Hz}$, $2 \mathrm{H}$, aromatic); ${ }^{13} \mathrm{C}$ NMR (methanol- $\left.\mathrm{d}_{4}\right) \delta(\mathrm{ppm}) 18.3\left(\mathrm{CH}_{3}\right), 41.9\left(\mathrm{CH}_{2}\right), 68.5(\mathrm{CH}), 123.9$ $(\mathrm{CH}), 126.9(\mathrm{CH}), 135.3(\mathrm{C}), 139.1(\mathrm{C}), 171.9(\mathrm{CO}) ;[\alpha]^{22} \mathrm{D}+18.6\left(\mathrm{c} 0.66, \mathrm{CH}_{3} \mathrm{OH}\right)$.

(R)-3-(4'-Cyanophenyl)-3-hydroxypropanenitrile (2g). ${ }^{6}$ light yellow solid, $155 \mathrm{mg}$, 90\% yield; m.p. 80-82 ${ }^{\circ} \mathrm{C} .{ }^{1} \mathrm{H}$ NMR $\left(\mathrm{CDCl}_{3}\right) \delta(\mathrm{ppm}) 2.77-2.81\left(\mathrm{~m}, 2 \mathrm{H}, \mathrm{CH}_{2}\right), 2.97$ (brs, $1 \mathrm{H}, \mathrm{OH}), 5.11-5.14(\mathrm{~m}, 1 \mathrm{H}, \mathrm{CH}), 7.54(\mathrm{~d}, J=8.1 \mathrm{~Hz}, 2 \mathrm{H}$, aromatic), $7.70(\mathrm{~d}, J=6.6 \mathrm{~Hz}$, $2 \mathrm{H}$, aromatic); ${ }^{13} \mathrm{C}$ NMR $\left(\mathrm{CDCl}_{3}\right) \delta(\mathrm{ppm}) 27.9\left(\mathrm{CH}_{2}\right), 69.2(\mathrm{CH}), 112.5(\mathrm{CN}), 116.5$ $(\mathrm{CN}), 118.2(\mathrm{C}), 126.3(\mathrm{CH}), 132.6(\mathrm{CH}), 145.9(\mathrm{C}) ;[\alpha]^{22}{ }_{\mathrm{D}}+66.5\left(\mathrm{c} 1.67, \mathrm{CH}_{3} \mathrm{OH}\right)$.

(R)-3-(4'-Carboxyphenyl)-3-hydroxypropanoic acid $(\mathbf{4 g}) .{ }^{10}$ white solid, $68 \mathrm{mg}, 80 \%$ yield; ${ }^{1} \mathrm{H}$ NMR (methanol-d $\left.\mathrm{d}_{4}\right) \delta(\mathrm{ppm}) 2.67\left(\mathrm{~d}, J=7.8 \mathrm{~Hz}, 2 \mathrm{H}, \mathrm{CH}_{2}\right), 5.15(\mathrm{~m}, 1 \mathrm{H}, \mathrm{CH})$, $7.40(\mathrm{~d}, J=8.0 \mathrm{~Hz}, 2 \mathrm{H}$, aromatic $), 7.90\left(\mathrm{~d}, J=8.0 \mathrm{~Hz}, 2 \mathrm{H}\right.$, aromatic); ${ }^{13} \mathrm{C}$ NMR $($ methanol-d 4$) \delta(\mathrm{ppm}) 42.4\left(\mathrm{CH}_{2}\right), 68.7(\mathrm{CH}), 124.4(\mathrm{CH}), 128.3(\mathrm{CH}), 128.6(\mathrm{C}), 148.1$ (C), $167.2(\mathrm{CO}), 172.2(\mathrm{CO}) ;[\alpha]^{22}+95.36\left(\mathrm{c} 0.5, \mathrm{CH}_{3} \mathrm{OH}\right)$.

(R)-3-(4'-Cyanophenyl)-3-hydroxypropanoic acid (3g). ${ }^{10}$ light yellow solid, $80 \mathrm{mg}$, 90\% yield; ${ }^{1} \mathrm{H}$ NMR $\left(\mathrm{CDCl}_{3}\right) \delta(\mathrm{ppm}) 2.69\left(\mathrm{~d}, J=8.0 \mathrm{~Hz}, 2 \mathrm{H}, \mathrm{CH}_{2}\right), 5.13-5.16(\mathrm{~m}, 1 \mathrm{H}$, $\mathrm{CH}), 7.49\left(\mathrm{~d}, J=8.0 \mathrm{~Hz}, 2 \mathrm{H}\right.$, aromatic), $7.98\left(\mathrm{~d}, J=8.0 \mathrm{~Hz}, 2 \mathrm{H}\right.$, aromatic); ${ }^{13} \mathrm{C}$ NMR (acetone- $\left.\mathrm{d}_{6}\right) \delta(\mathrm{ppm}) 42.2\left(\mathrm{CH}_{2}\right), 68.4(\mathrm{CH}), 109.6(\mathrm{CN}), 117.2(\mathrm{C}), 124.4(\mathrm{CH}), 125.4$ (C), $128.4(\mathrm{C}), 130.8(\mathrm{CH}), 148.6(\mathrm{C}), 171.8(\mathrm{CO}) ;[\alpha]^{22}{ }_{\mathrm{D}}+12.27\left(\mathrm{c} 0.33, \mathrm{CH}_{3} \mathrm{OH}\right)$.

(R)-3-Hydroxy-3-(4'-nitrophenyl)propanenitrile (2h). ${ }^{7}$ light yellow solid, $147 \mathrm{mg}$, $86 \%$ yield; m.p. $95-98^{\circ} \mathrm{C} .{ }^{1} \mathrm{H}$ NMR $\left(\mathrm{CDCl}_{3}\right) \delta(\mathrm{ppm})$ 2.75-2.85 (m, $\left.2 \mathrm{H}, \mathrm{CH}_{2}\right), 3.05$ (brs, $1 \mathrm{H}, \mathrm{OH}), 5.17-5.20(\mathrm{~m}, 1 \mathrm{H}, \mathrm{CH}), 7.61(\mathrm{~d}, J=8.8 \mathrm{~Hz}, 2 \mathrm{H}$, aromatic), $8.25(\mathrm{~d}, J=8.6 \mathrm{~Hz}$, $2 \mathrm{H}$, aromatic); ${ }^{13} \mathrm{C} \mathrm{NMR}\left(\mathrm{CDCl}_{3}\right) \delta(\mathrm{ppm}) 28.5\left(\mathrm{CH}_{2}\right), 69.4(\mathrm{CH}), 116.9(\mathrm{CN}), 124.5$ $(\mathrm{CH}), 127.0(\mathrm{CH}), 148.2(\mathrm{C}), 148.4(\mathrm{C}) ;[\alpha]^{22}{ }_{\mathrm{D}}+12.33\left(\mathrm{c} 0.50, \mathrm{CH}_{3} \mathrm{OH}\right)$.

(R)-3-Hydroxy-3-(4'-nitrophenyl)propanoic acid (3h). ${ }^{10}$ light yellow solid, $77 \mathrm{mg}$, $88 \%$ yield; ${ }^{1} \mathrm{H}$ NMR (methanol- $\left.\mathrm{d}_{4}\right) \delta(\mathrm{ppm}) 2.70-2.72\left(\mathrm{~m}, 2 \mathrm{H}, \mathrm{CH}_{2}\right), 5.20-5.22(\mathrm{~m}, 1 \mathrm{H}$, $\mathrm{CH}), 7.66\left(\mathrm{~d}, J=8.0 \mathrm{~Hz}, 1 \mathrm{H}\right.$, aromatic), $8.23\left(\mathrm{~d}, J=8.0 \mathrm{~Hz}, 1 \mathrm{H}\right.$, aromatic); ${ }^{13} \mathrm{C}$ NMR $($ methanol-d 4$) \delta(\mathrm{ppm}) 42.3\left(\mathrm{CH}_{2}\right), 68.3(\mathrm{CH}), 121.9(\mathrm{CH}), 125.5(\mathrm{CH}), 146.2(\mathrm{C}), 150.5$ (C), $171.9(\mathrm{CO}) ;[\alpha]^{22}+28.9\left(\mathrm{c} 0.5, \mathrm{CH}_{3} \mathrm{OH}\right)$.

(R)-3-Hydroxy-3-(3'-nitrophenyl)propanenitrile (2i). ${ }^{7}$ light yellow solid, $150 \mathrm{mg}, 87 \%$ yield; m.p. $86-89^{\circ} \mathrm{C} ;{ }^{1} \mathrm{H}$ NMR $\left(\mathrm{CDCl}_{3}\right) \delta(\mathrm{ppm}) 2.81-2.83\left(\mathrm{~m}, 2 \mathrm{H}, \mathrm{CH}_{2}\right), 3.93$ (brs, $1 \mathrm{H}$, $\mathrm{OH}), 5.18(\mathrm{br}, 1 \mathrm{H}, \mathrm{CH}), 7.59(\mathrm{t}, J=8.0 \mathrm{~Hz}, 1 \mathrm{H}$, aromatic), $7.76(\mathrm{~d}, J=8.0 \mathrm{~Hz}, 1 \mathrm{H}$, aromatic), $8.20\left(\mathrm{~d}, J=8.0 \mathrm{~Hz}, 1 \mathrm{H}\right.$, aromatic), $8.30\left(\mathrm{~s}, 1 \mathrm{H}\right.$, aromatic); ${ }^{13} \mathrm{C} \mathrm{NMR}\left(\mathrm{CDCl}_{3}\right) \delta$ (ppm) $28.1\left(\mathrm{CH}_{2}\right), 68.7(\mathrm{CH}), 116.7(\mathrm{CN}), 120.7(\mathrm{CH}), 123.5(\mathrm{CH}), 129.8(\mathrm{CH}), 131.7$ $(\mathrm{CH}), 143.4(\mathrm{C}), 148.4(\mathrm{C}) ;[\alpha]_{\mathrm{D}}^{22}+22.3\left(\mathrm{c} 1.67, \mathrm{CH}_{3} \mathrm{OH}\right)$. 
(R)-3-Hydroxy-3-(3'-nitrophenyl)propanoic acid (3i). ${ }^{10}$ light yellow solid, $76 \mathrm{mg}$, $87 \%$ yield; m.p. $110-113^{\circ} \mathrm{C}$. ${ }^{1} \mathrm{H}$ NMR (methanol-d 4 ) $\delta(\mathrm{ppm}) 2.72-2.74(\mathrm{~d}, J=8.1 \mathrm{~Hz}$, $2 \mathrm{H}, \mathrm{CH}_{2}$ ), $5.20-5.23$ (t, $\left.J=7.6 \mathrm{~Hz}, 1 \mathrm{H}, \mathrm{CH}\right), 7.59$ (t, $J=7.7 \mathrm{~Hz}, 1 \mathrm{H}$, aromatic), 7.80 (d, $J$ $=8.2 \mathrm{~Hz}, 1 \mathrm{H}$, aromatic $), 8.14(\mathrm{~d}, J=8.2 \mathrm{~Hz}, 1 \mathrm{H}$, aromatic $), 8.30(\mathrm{~s}, 1 \mathrm{H}$, aromatic $) ;{ }^{13} \mathrm{C}$ NMR (methanol-d 4$) \delta(\mathrm{ppm}) 46.2\left(\mathrm{CH}_{2}\right), 72.0(\mathrm{CH}), 123.2(\mathrm{CH}), 124.7(\mathrm{CH}), 132.0$ $(\mathrm{CH}), 134.7(\mathrm{CH}), 149.3(\mathrm{C}), 151.2(\mathrm{C}), 175.8(\mathrm{CO}) ;[\alpha]^{22}+7.7\left(\mathrm{c} 0.5, \mathrm{CH}_{3} \mathrm{OH}\right)$.

(R)-3-Hydroxy-3-(3'-methoxyphenyl)propanenitrile (2j). ${ }^{1}$ viscous oil, $153 \mathrm{mg}, 89 \%$ yield; ${ }^{1} \mathrm{H}$ NMR $\left(\mathrm{CDCl}_{3}\right) \delta(\mathrm{ppm}) 2.68-2.71\left(\mathrm{~m}, 2 \mathrm{H}, \mathrm{CH}_{2}\right), 3.33$ (brs, $\left.1 \mathrm{H}, \mathrm{OH}\right), 3.79(\mathrm{~s}$, $\left.3 \mathrm{H}, \mathrm{OCH}_{3}\right), 4.93(\mathrm{br}, 1 \mathrm{H}, \mathrm{CH}), 6.84(\mathrm{~d}, J=8.0 \mathrm{~Hz}, 2 \mathrm{H}$, aromatic), $6.92(\mathrm{~s}, 1 \mathrm{H}$, aromatic) $7.27\left(\mathrm{~d}, J=8.0 \mathrm{~Hz}, 2 \mathrm{H}\right.$, aromatic) ${ }^{13}{ }^{3} \mathrm{NMR}\left(\mathrm{CDCl}_{3}\right) \delta(\mathrm{ppm}) 27.3\left(\mathrm{CH}_{2}\right), 55.2\left(\mathrm{OCH}_{3}\right)$, $69.6(\mathrm{CH}), 111.0(\mathrm{CH}), 113.9(\mathrm{CH}), 117.4(\mathrm{CN}), 117.7(\mathrm{CH}), 129.8(\mathrm{CH}), 142.7(\mathrm{C})$, $159.7(\mathrm{C}) ;[\alpha]_{\mathrm{D}}^{22}+26.5\left(\mathrm{c} 1.67, \mathrm{CH}_{3} \mathrm{OH}\right)$.

(R)-3-Hydroxy-3-(3'-methoxyphenyl)propanoic acid (3j). ${ }^{1}$ white crystalline solid, 61 mg, $90 \%$ yield; m.p. $110-112^{\circ} \mathrm{C} .{ }^{1} \mathrm{H}$ NMR (methanol- $\left.\mathrm{d}_{4}\right) \delta(\mathrm{ppm}) 2.54-2.65(\mathrm{~m}, 2 \mathrm{H}$, $\left.\mathrm{CH}_{2}\right), 3.71\left(\mathrm{~s}, 3 \mathrm{H}, \mathrm{OCH}_{3}\right), 4.99-5.02(\mathrm{~m}, 1 \mathrm{H}, \mathrm{CH}), 6.74(\mathrm{~s}, 1 \mathrm{H}$, aromatic), $6.88(\mathrm{~d}, J=8.1$ $\mathrm{Hz}, 2 \mathrm{H}$, aromatic), $7.15\left(\mathrm{t}, J=8.1 \mathrm{~Hz}, 1 \mathrm{H}\right.$, aromatic); ${ }^{13} \mathrm{C}$ NMR (methanol-d $\left.\mathrm{d}_{4}\right) \delta(\mathrm{ppm})$ $42.5\left(\mathrm{CH}_{2}\right), 53.0\left(\mathrm{OCH}_{3}\right), 69.1(\mathrm{CH}), 109.8(\mathrm{CH}), 111.5(\mathrm{CH}), 116.5(\mathrm{CH}), 127.7(\mathrm{CH})$, $144.3(\mathrm{C}), 158.7(\mathrm{C}), 172.4(\mathrm{CO}) ;[\alpha]^{22} \mathrm{D}+17.7\left(\mathrm{c} 0.83, \mathrm{CH}_{3} \mathrm{OH}\right)$.

\section{References}

(1) Kamila, S.; Zhu, D.; Biehl, E. R.; Hua, L. Org. Lett. 2006, 8, 4429-4431.

(2) Zhu, D.; Yang, Y.; Hua, L. J. Org. Chem. 2006, 71, 4202-4205.

(3) Mukherjee, C.; Zhu, D.; Biehl, E. R.; Hua, L. Eur. J. Org. Chem. 2006, $5238-5242$.

(4) Zhu, D.; Mukherjee, C.; Biehl, E. R.; Hua, L. J. Biotechnol. 2007, 129, 645-650.

(5) Zhou, Z.; Shi, L.; Huang, Y. Z. Journal of the Chemical Society, Perkin Transactions 1: Organic and Bio-Organic Chemistry (1972-1999) 1991, 1931-3.

(6) Sun, P.; Shi, B. J. Chem. Res., Synop. 1999, 318-319.

(7) Araki, S.; Yamada, M.; Butsugan, Y. Bull. Chem. Soc. Jpn. 1994, 67, $1126-9$.

(8) Liu, P. N.; Gu, P. M.; Wang, F.; Tu, Y. Q. Org. Lett. 2004, 6, 169-172.

(9) Leclercq, M.; Collet, A.; Jacques, J. Tetrahedron 1976, 32, 821-8.

(10) Washio, T.; Nakamura, S.; Anada, M.; Hashimoto, S. Heterocycles 2005, $66,567-578$.

(11) Meisterhans, C.; Linden, A.; Hesse, M. Helv. Chim. Acta 2003, 86, 644656.

(12) Kamal, A.; Khanna, G. B. R.; Ramu, R. Tetrahedron: Asymmetry 2002, 13, 2039-2051.

6. $\quad{ }^{13} \mathrm{C}$ NMR spectra of $\beta$-hydroxy nitriles and $\beta$-hydroxy carboxylic acids. 


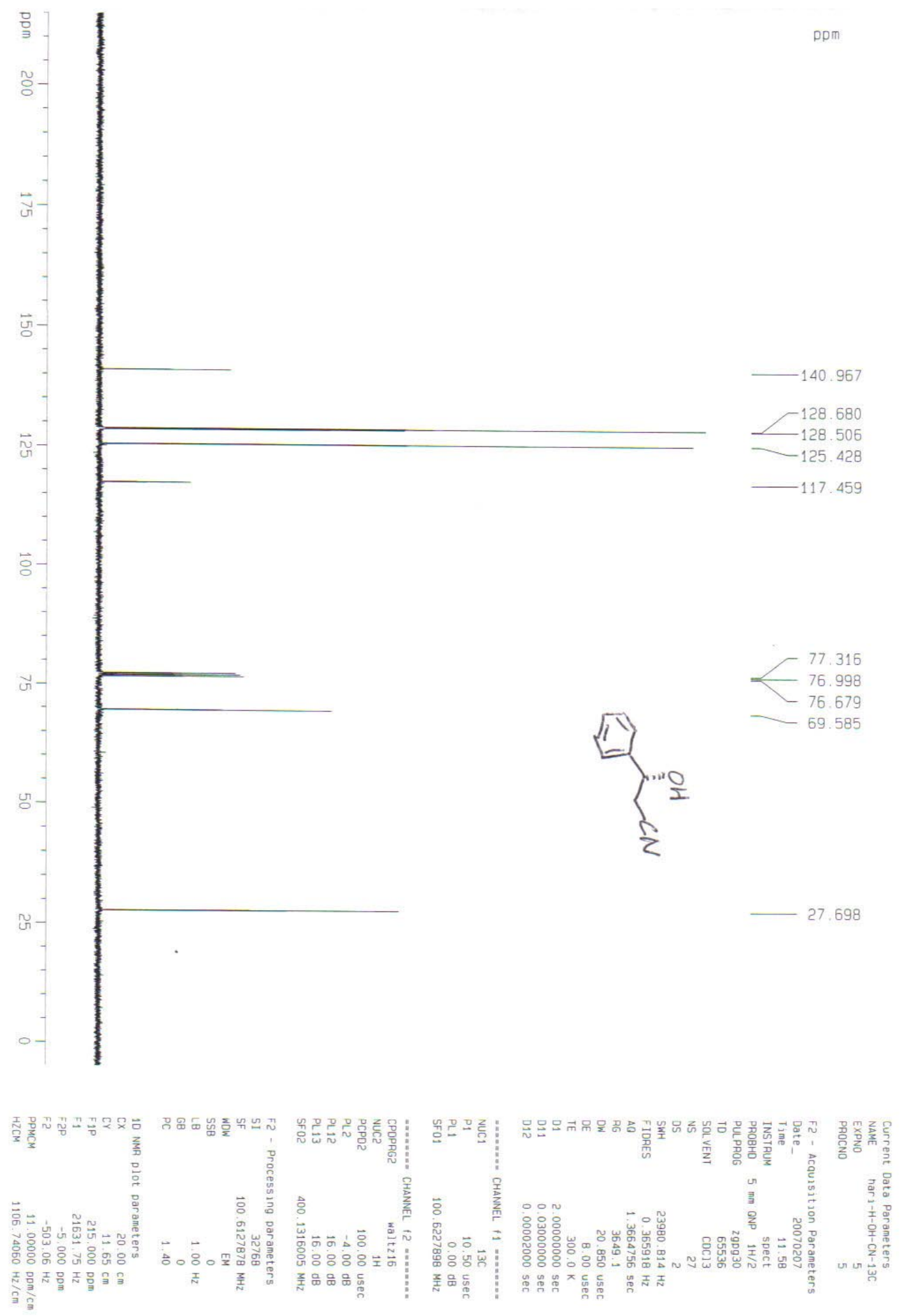




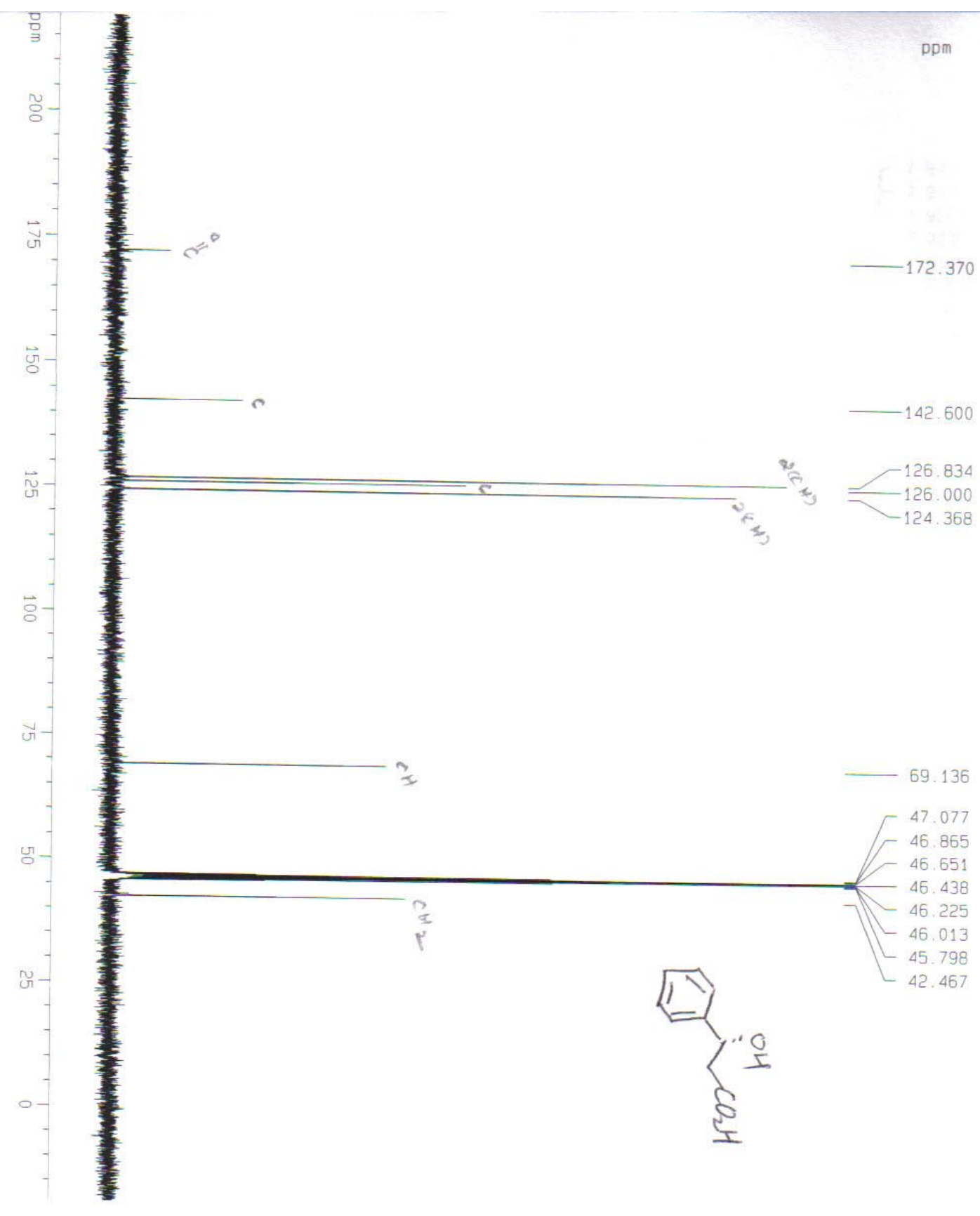

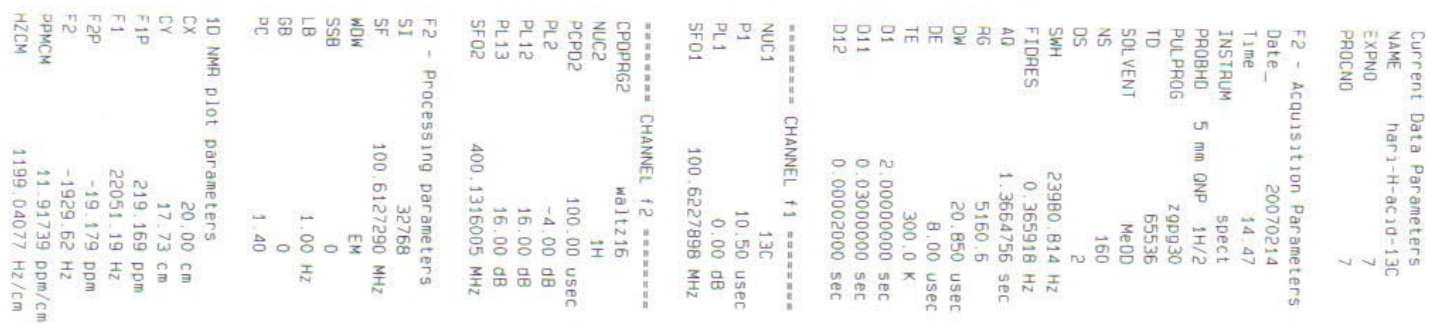




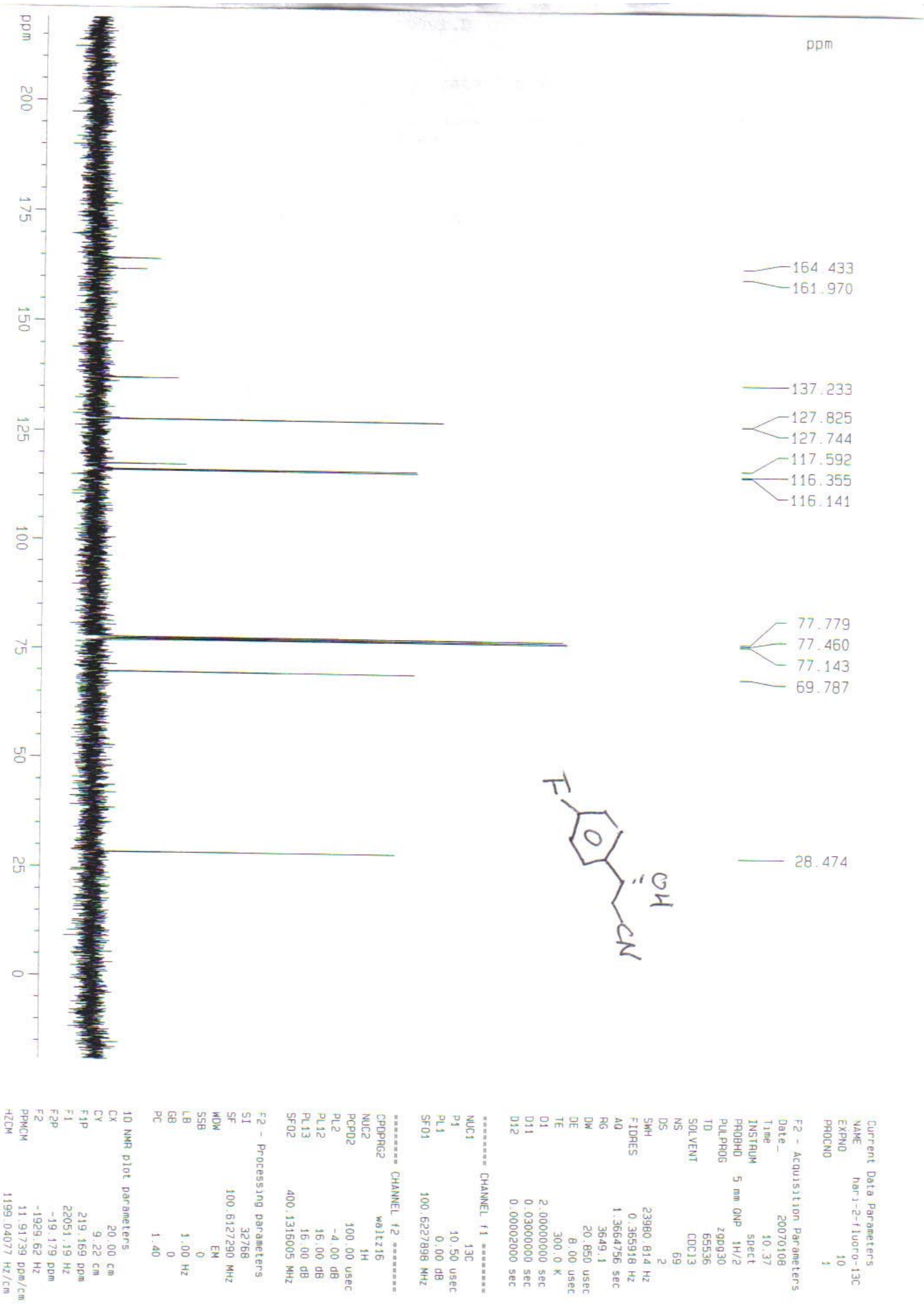



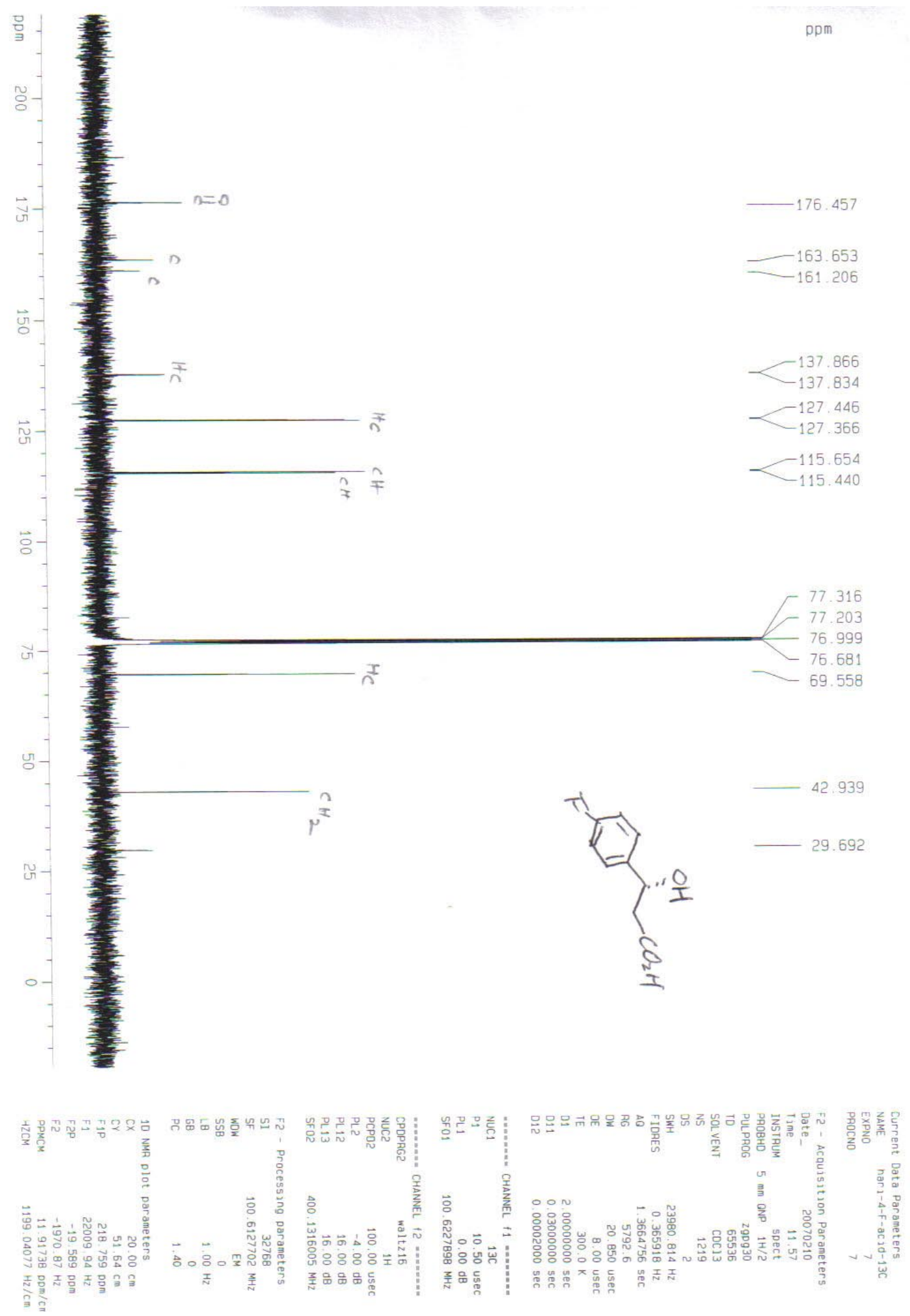


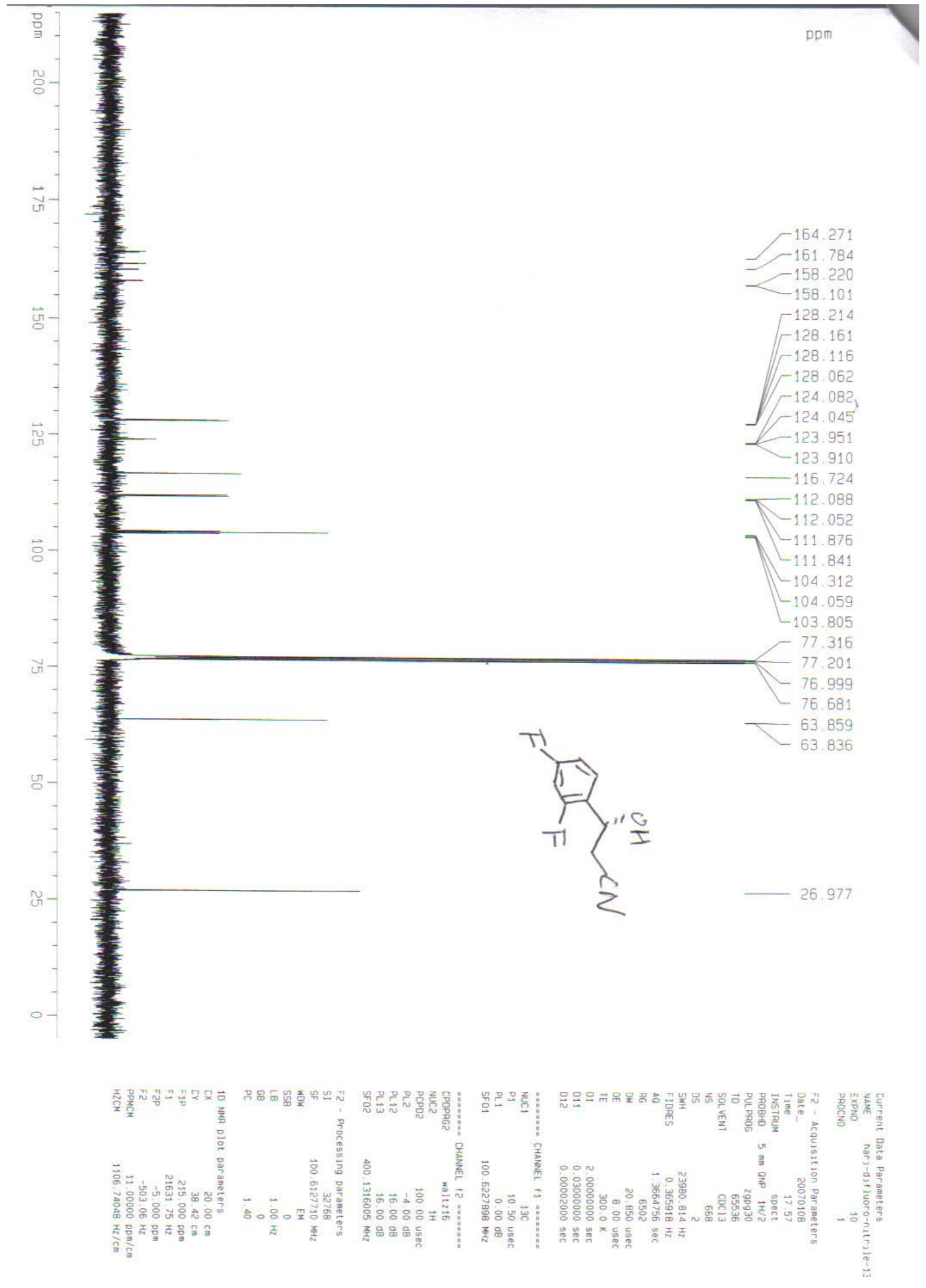




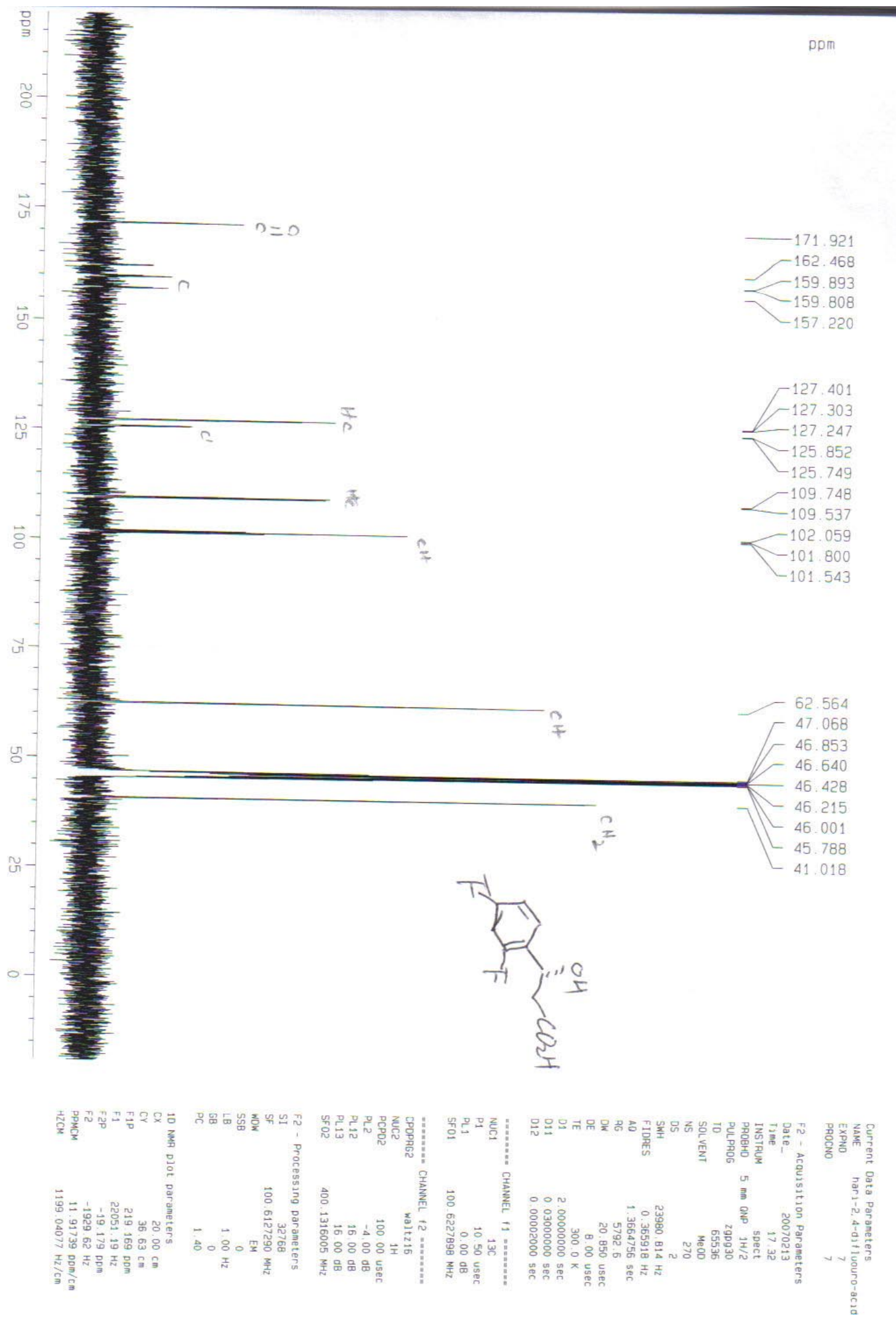



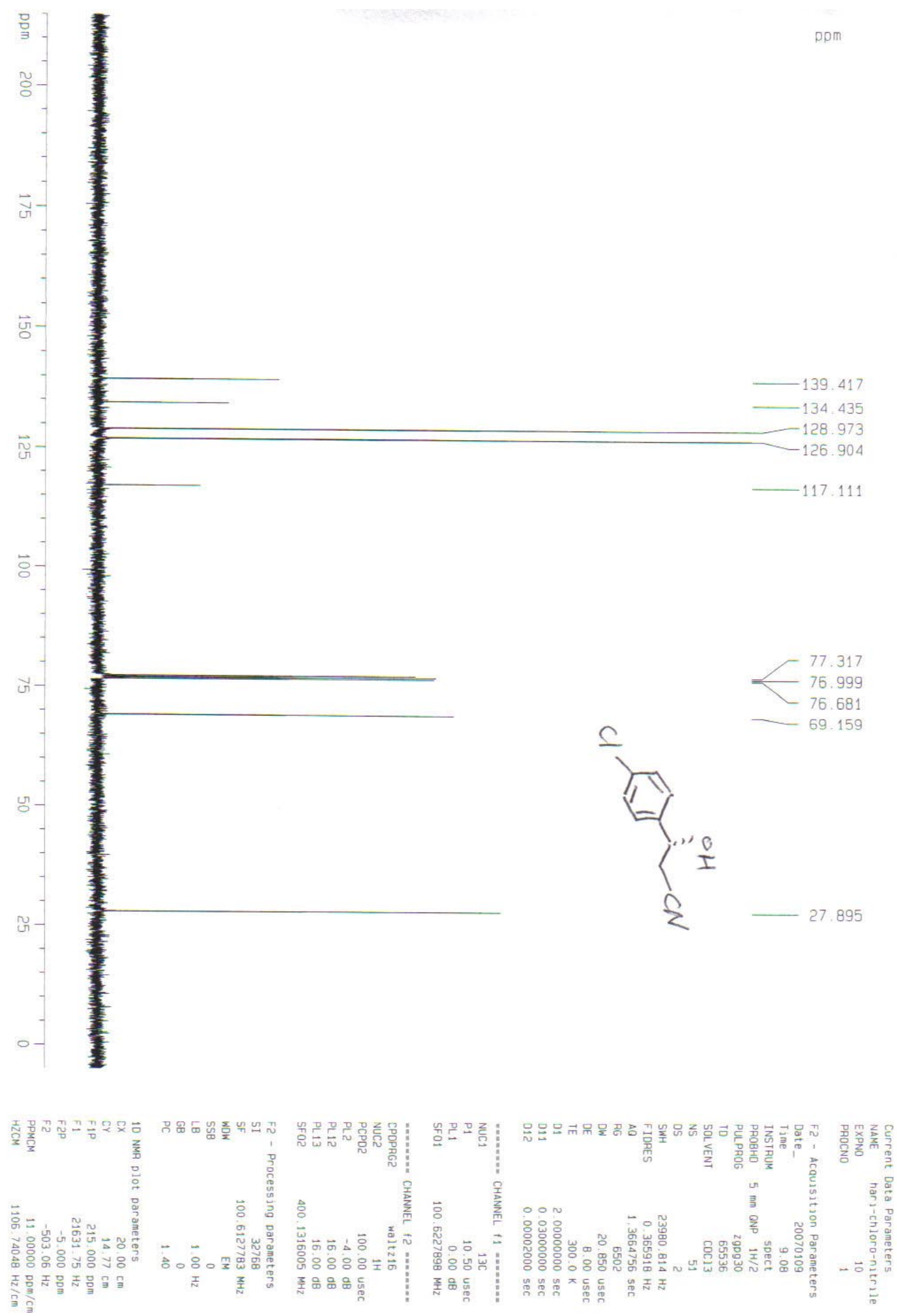


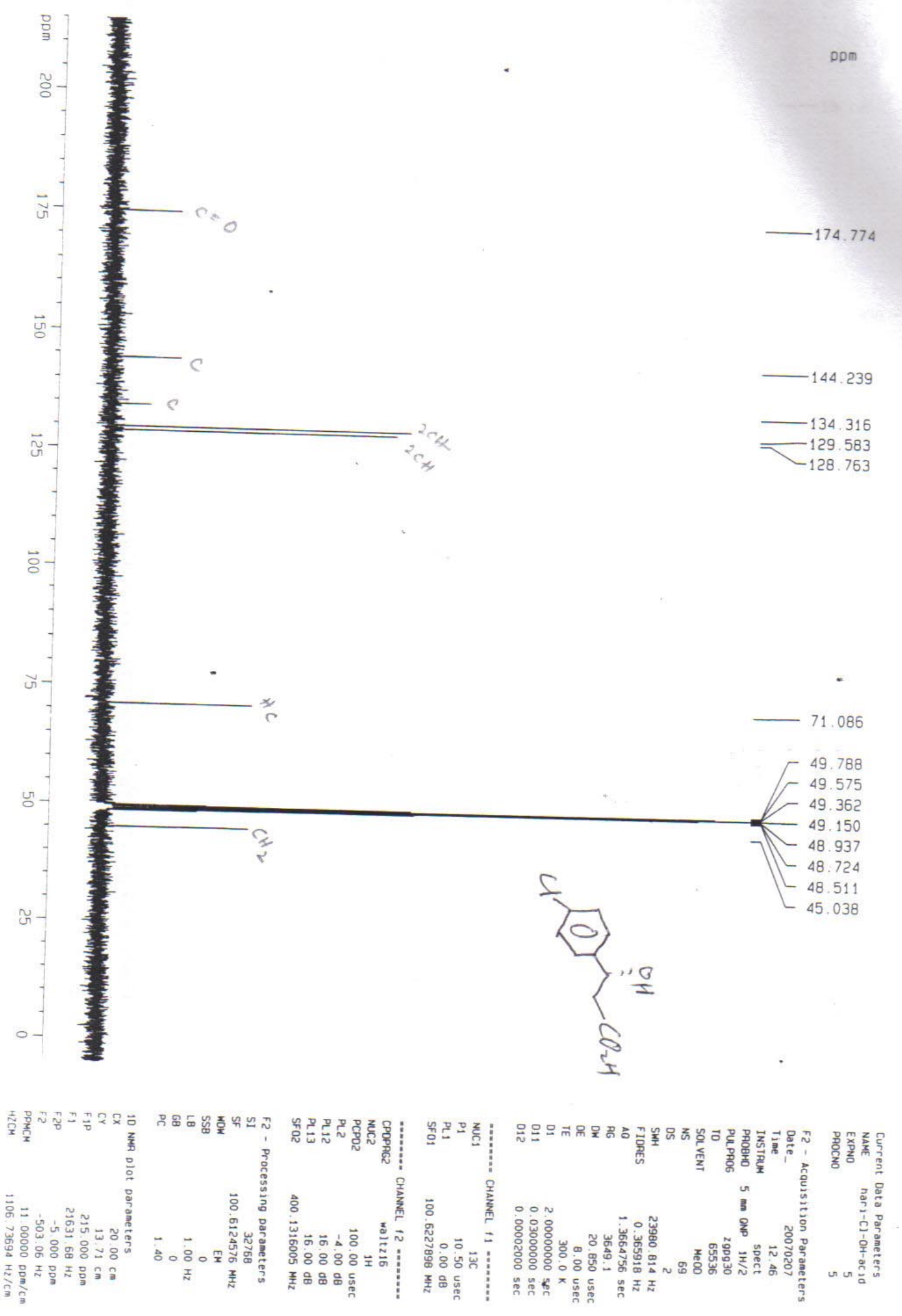




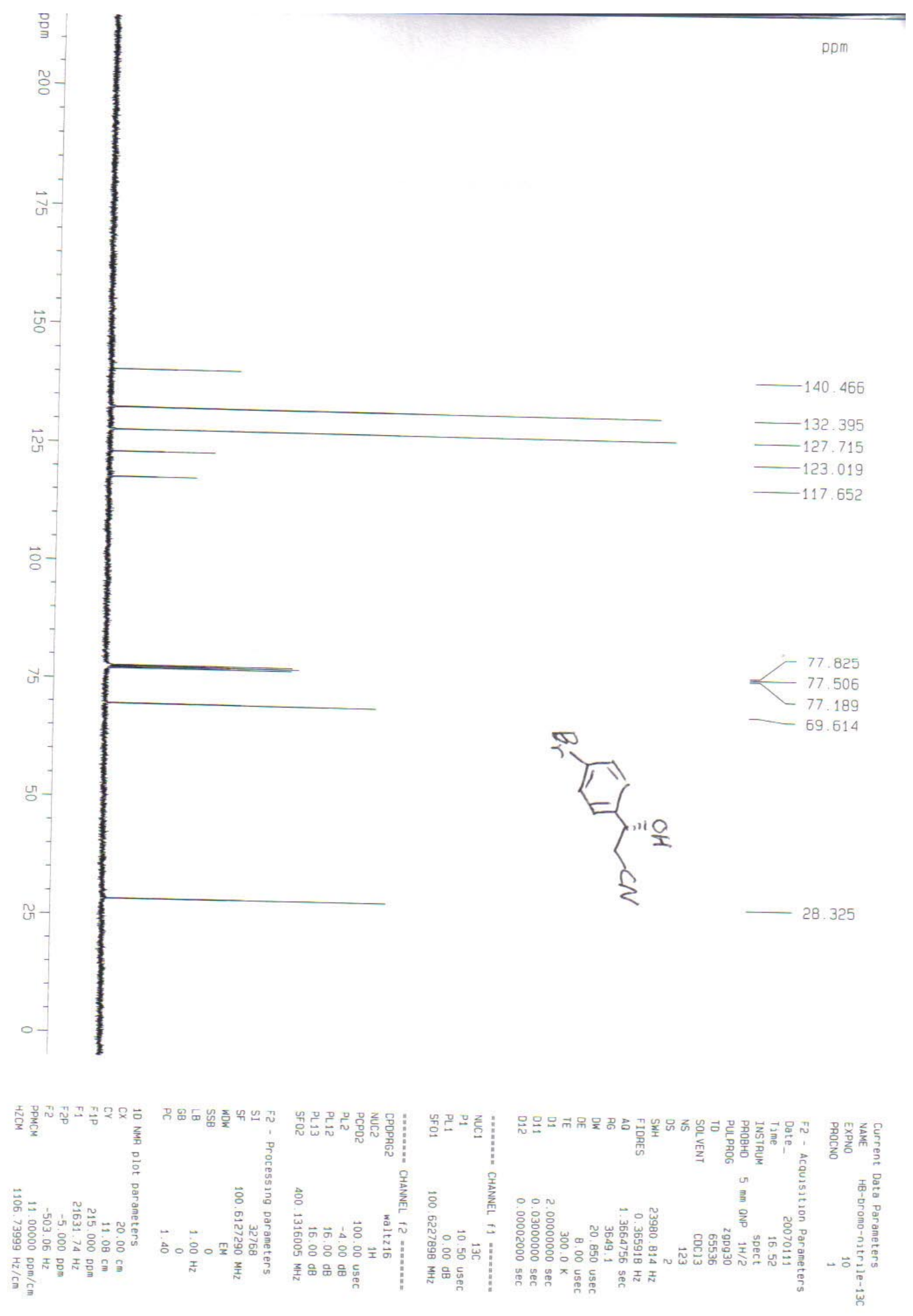




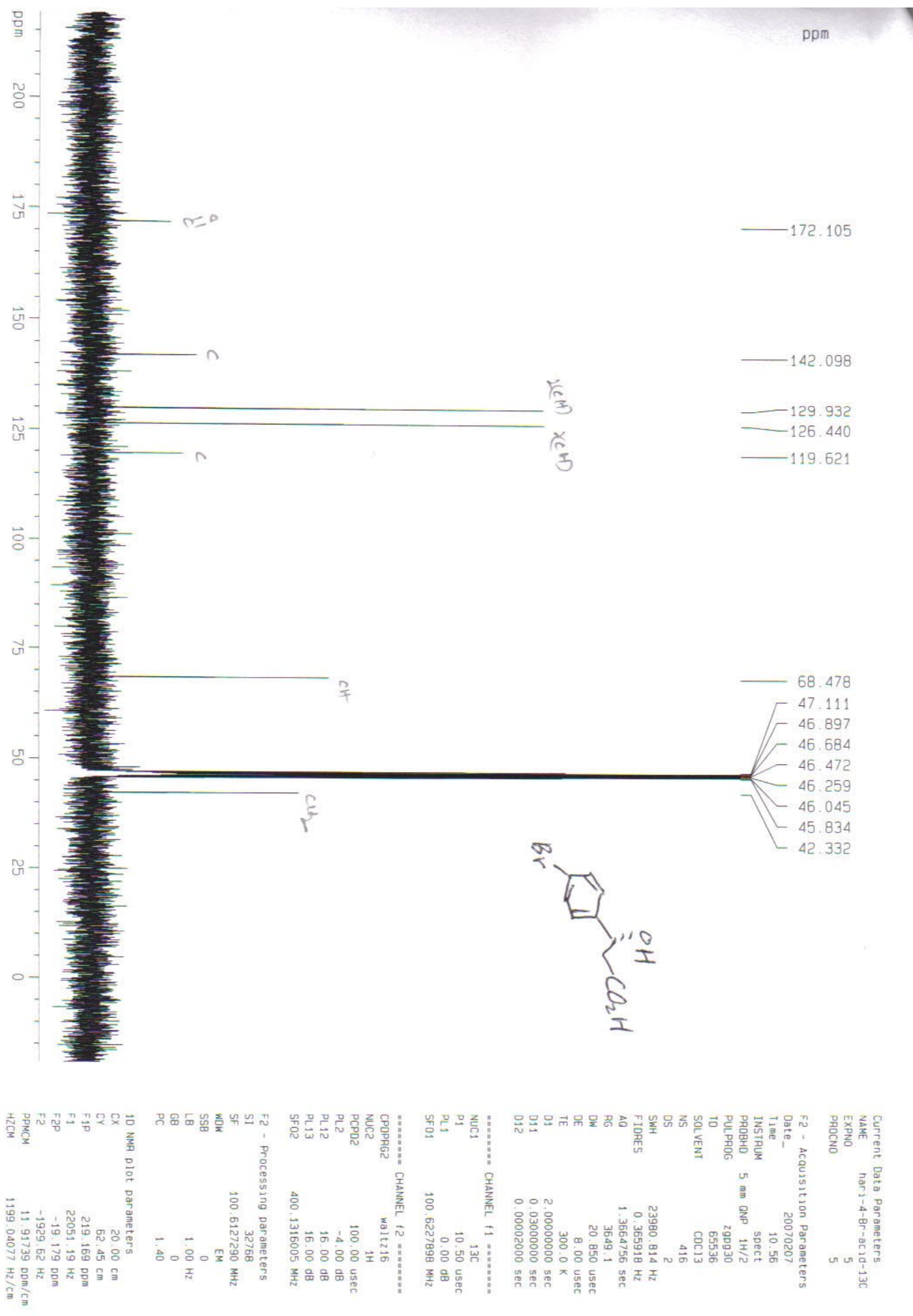




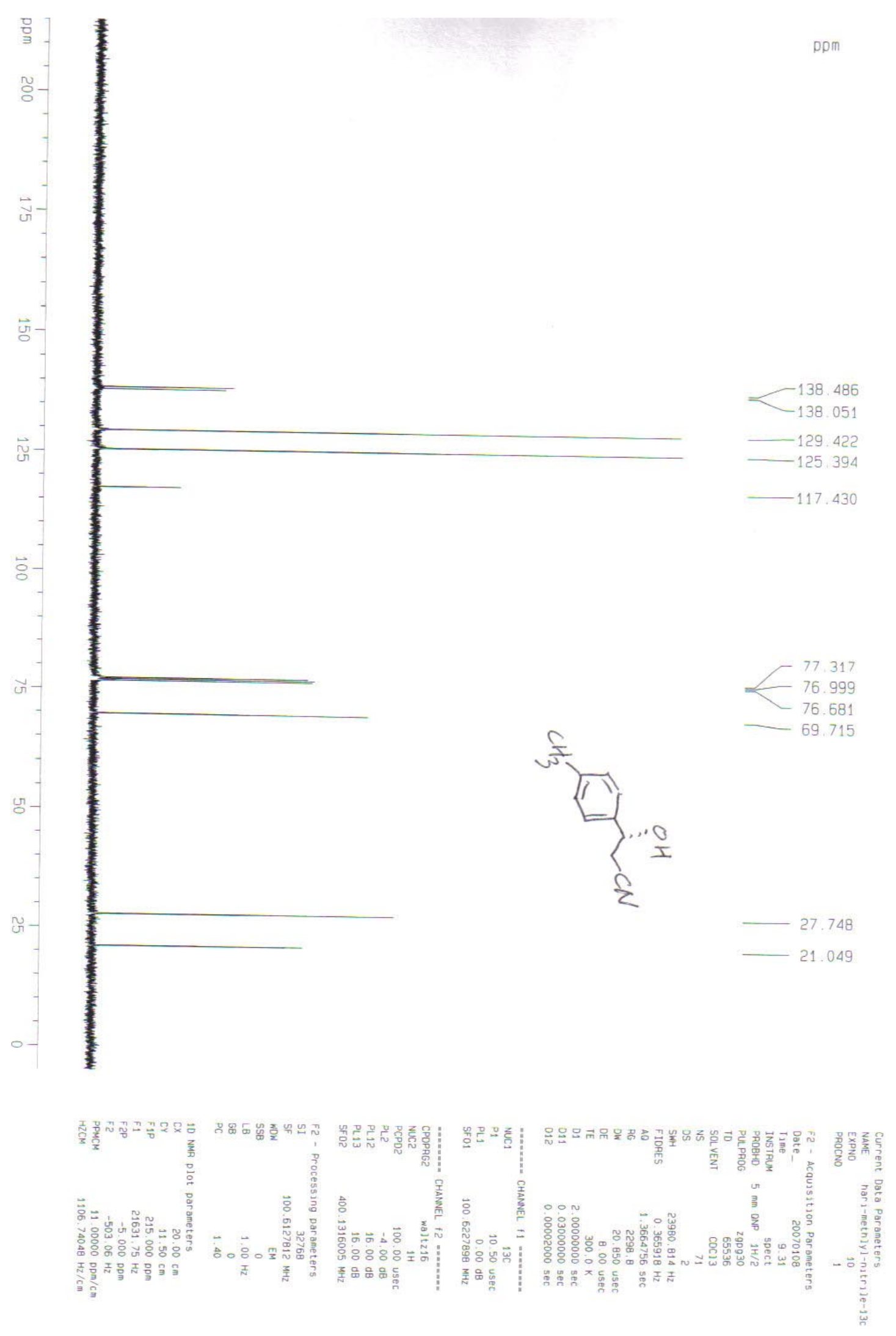




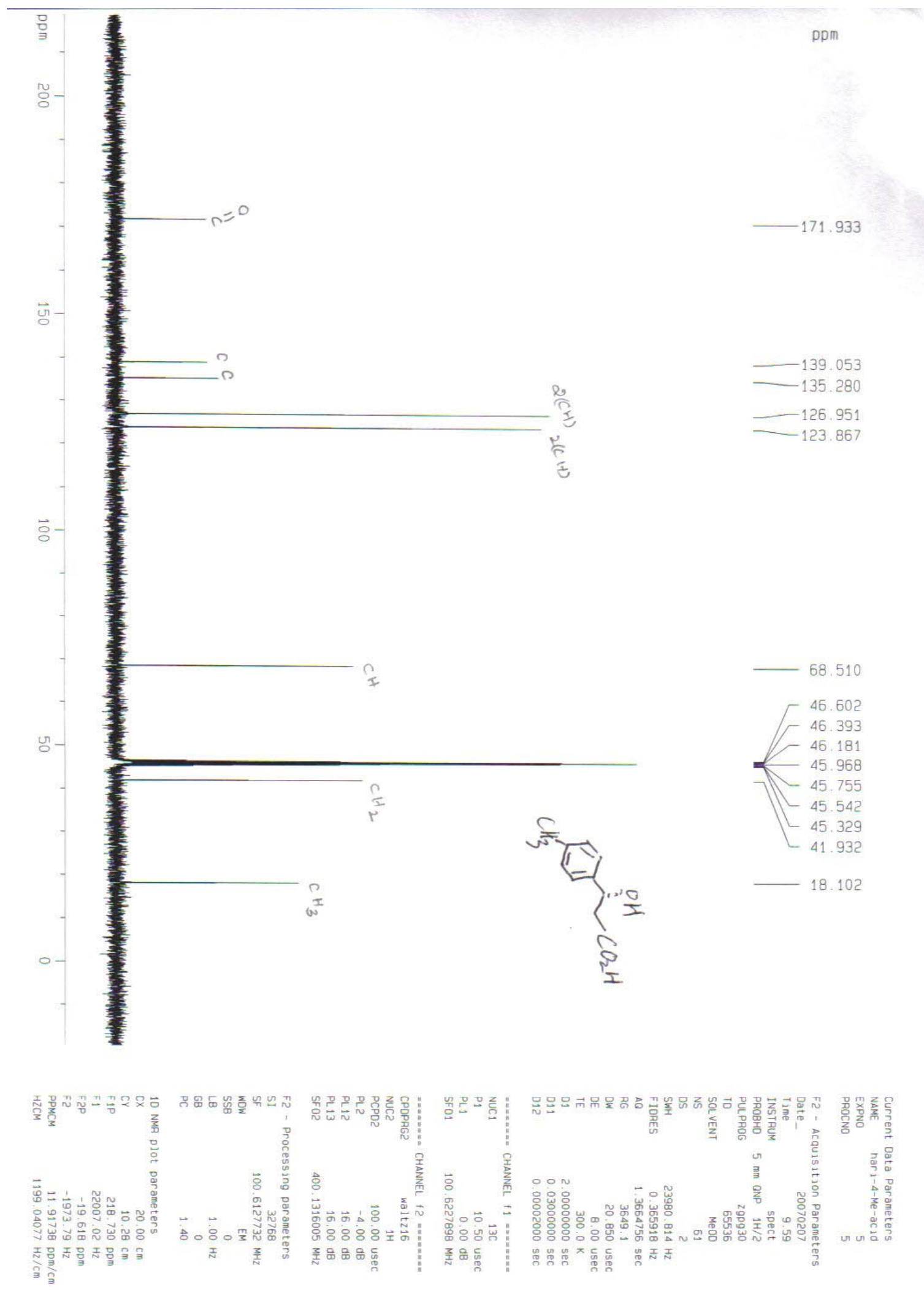




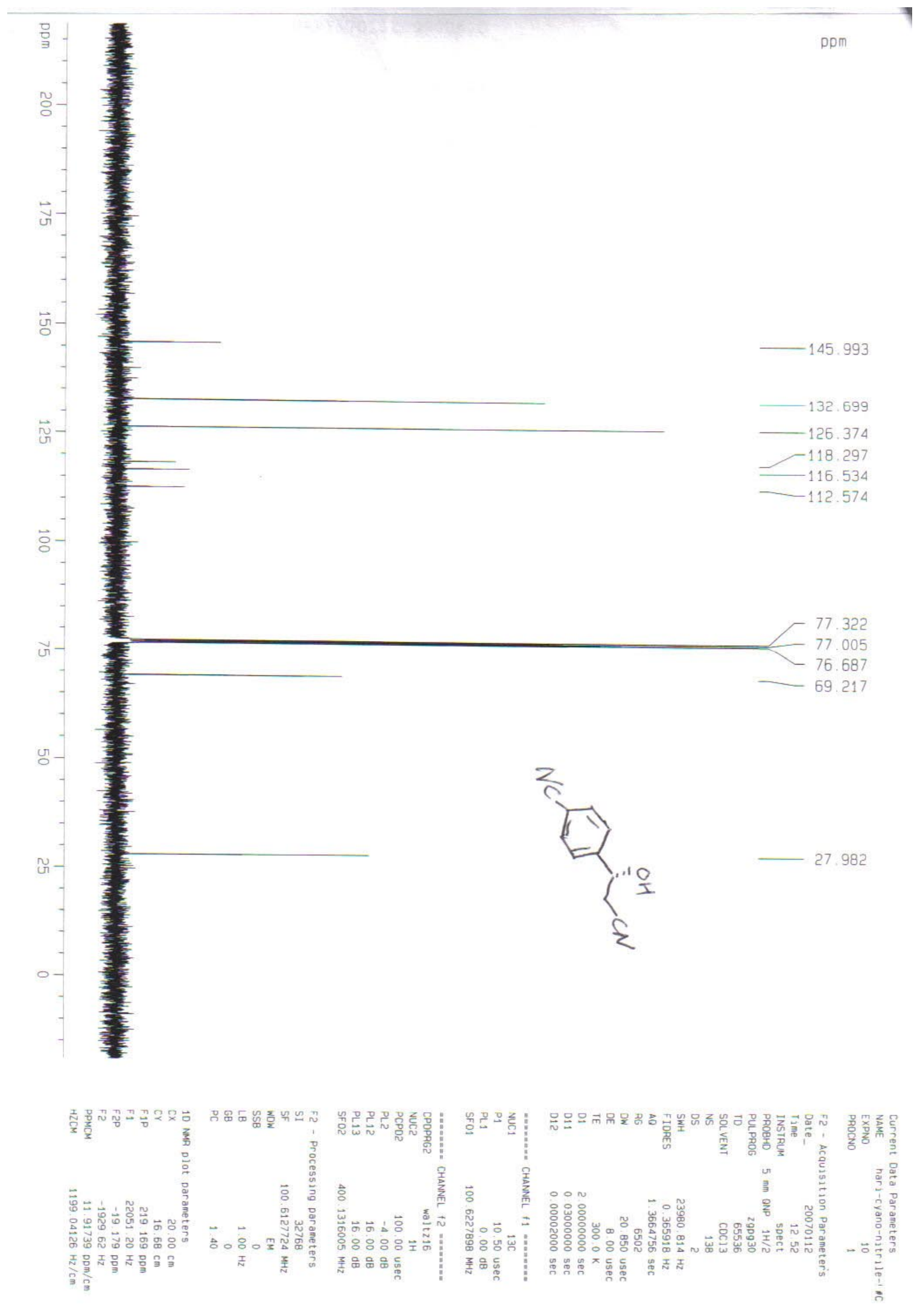



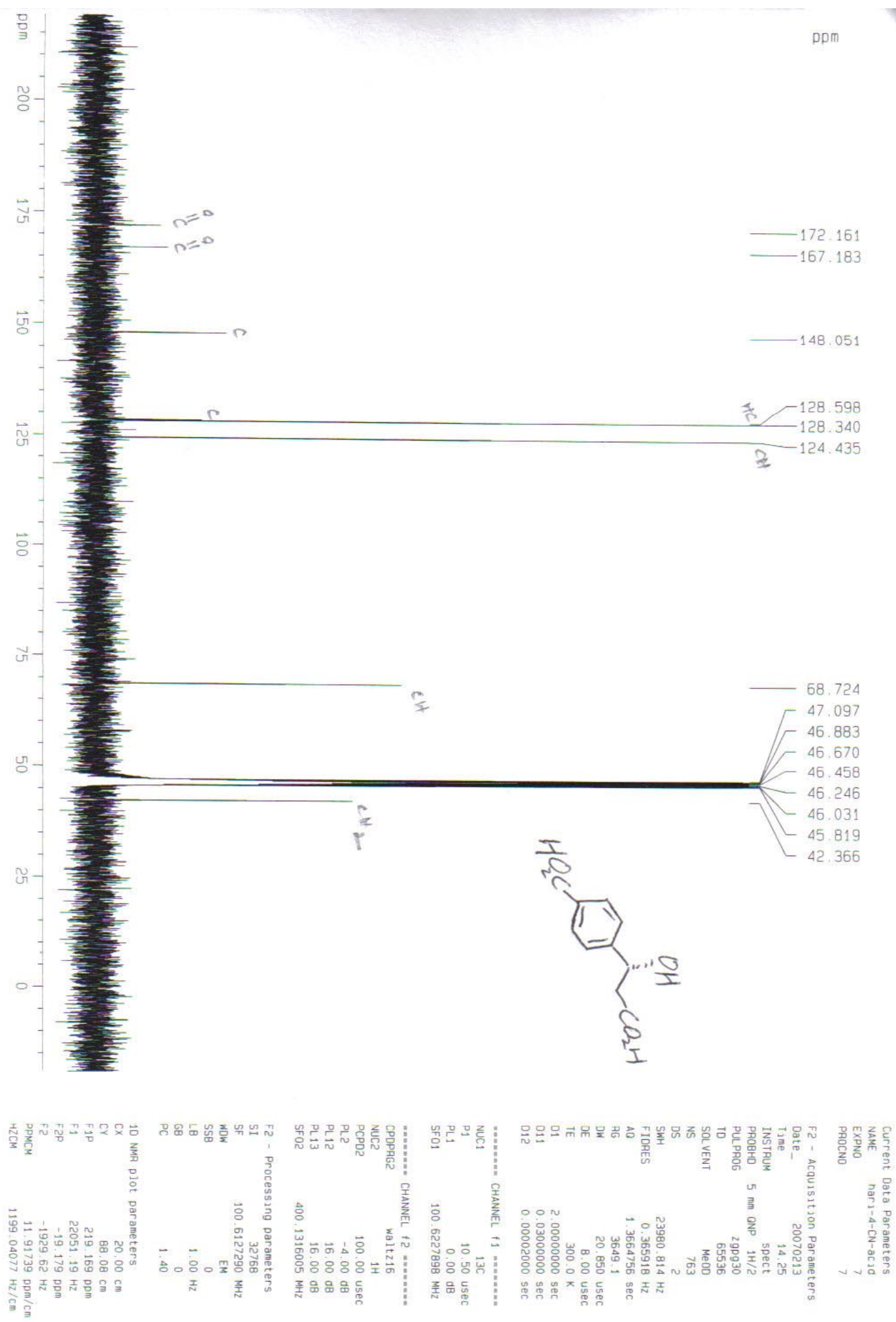


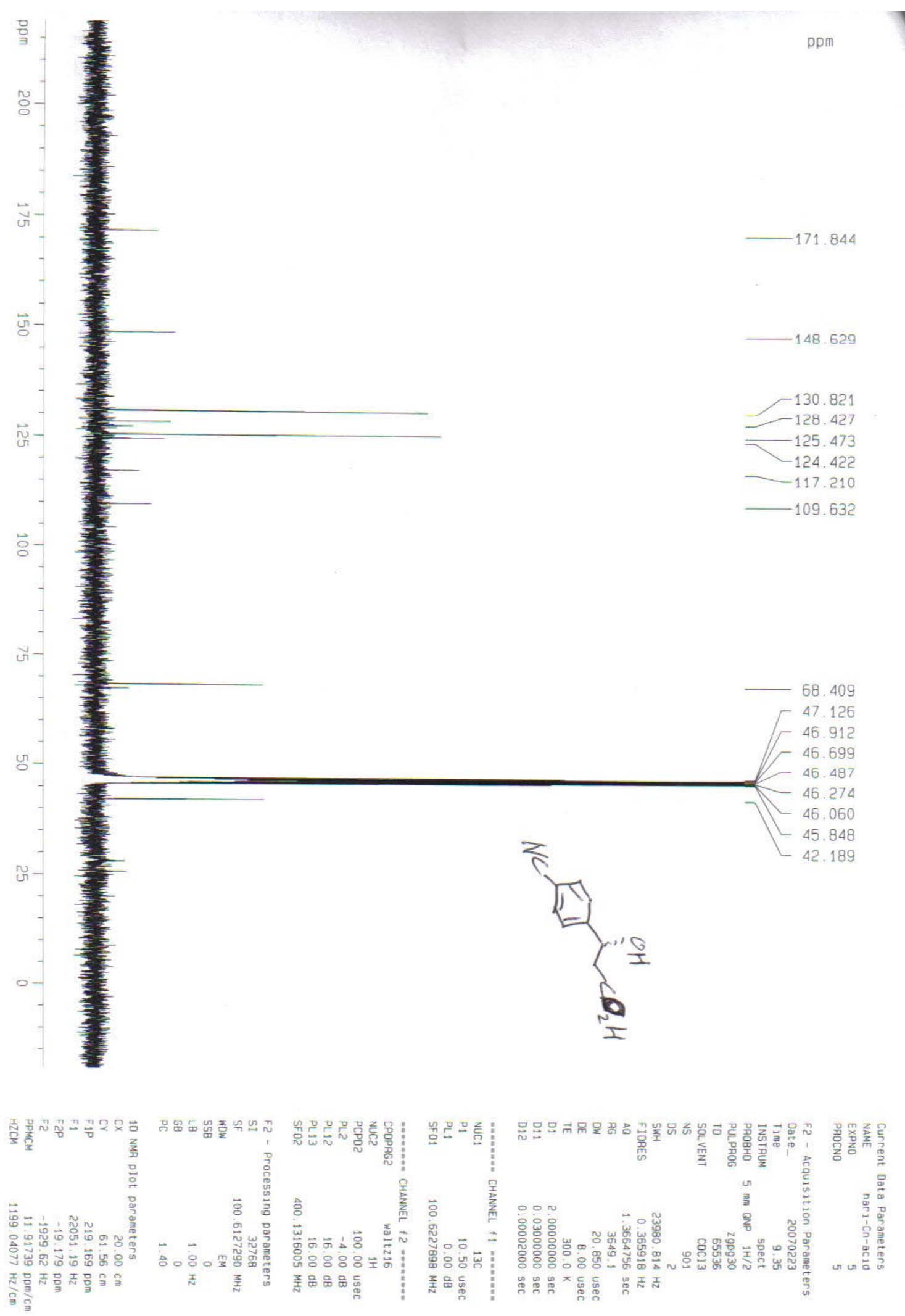



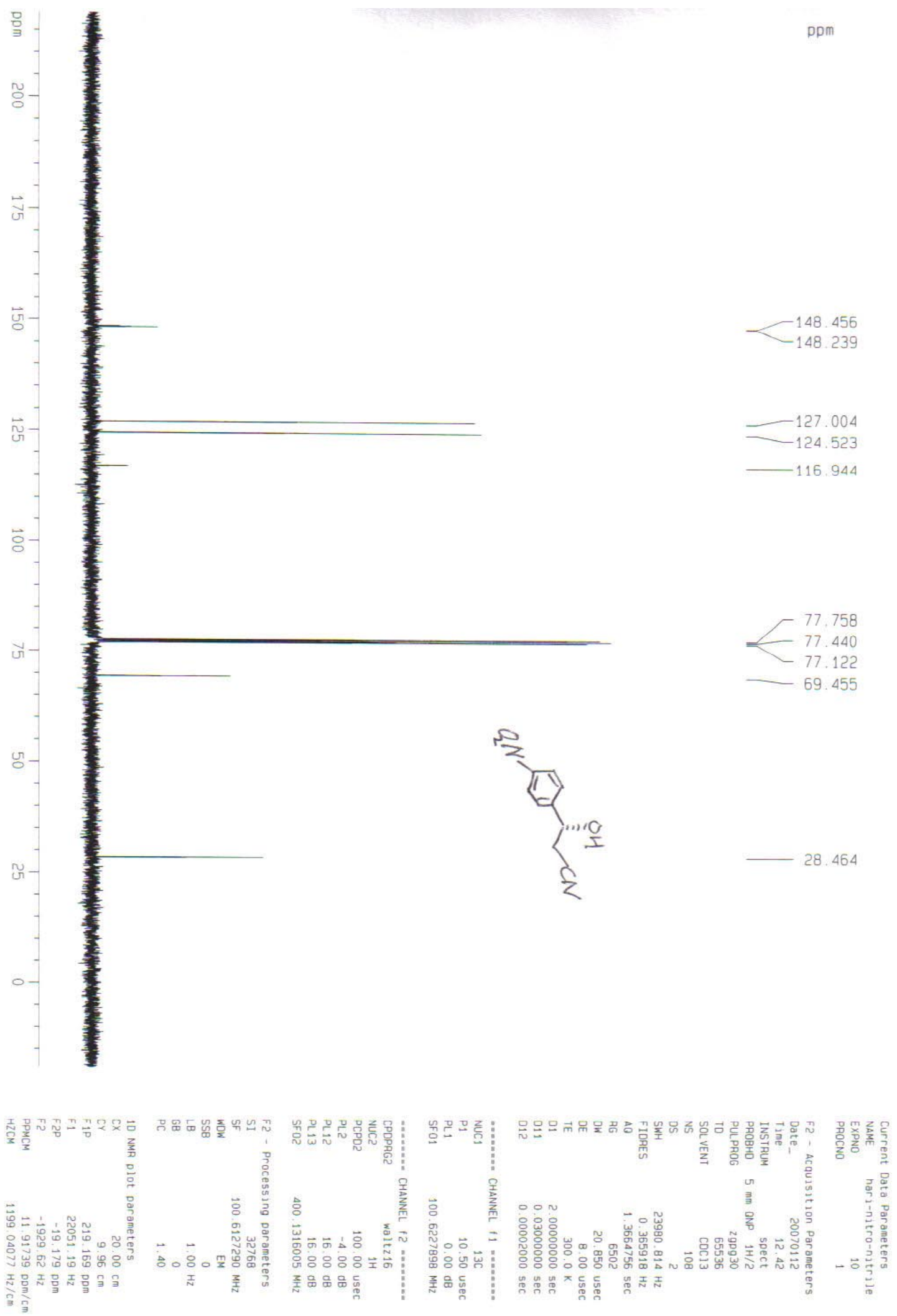


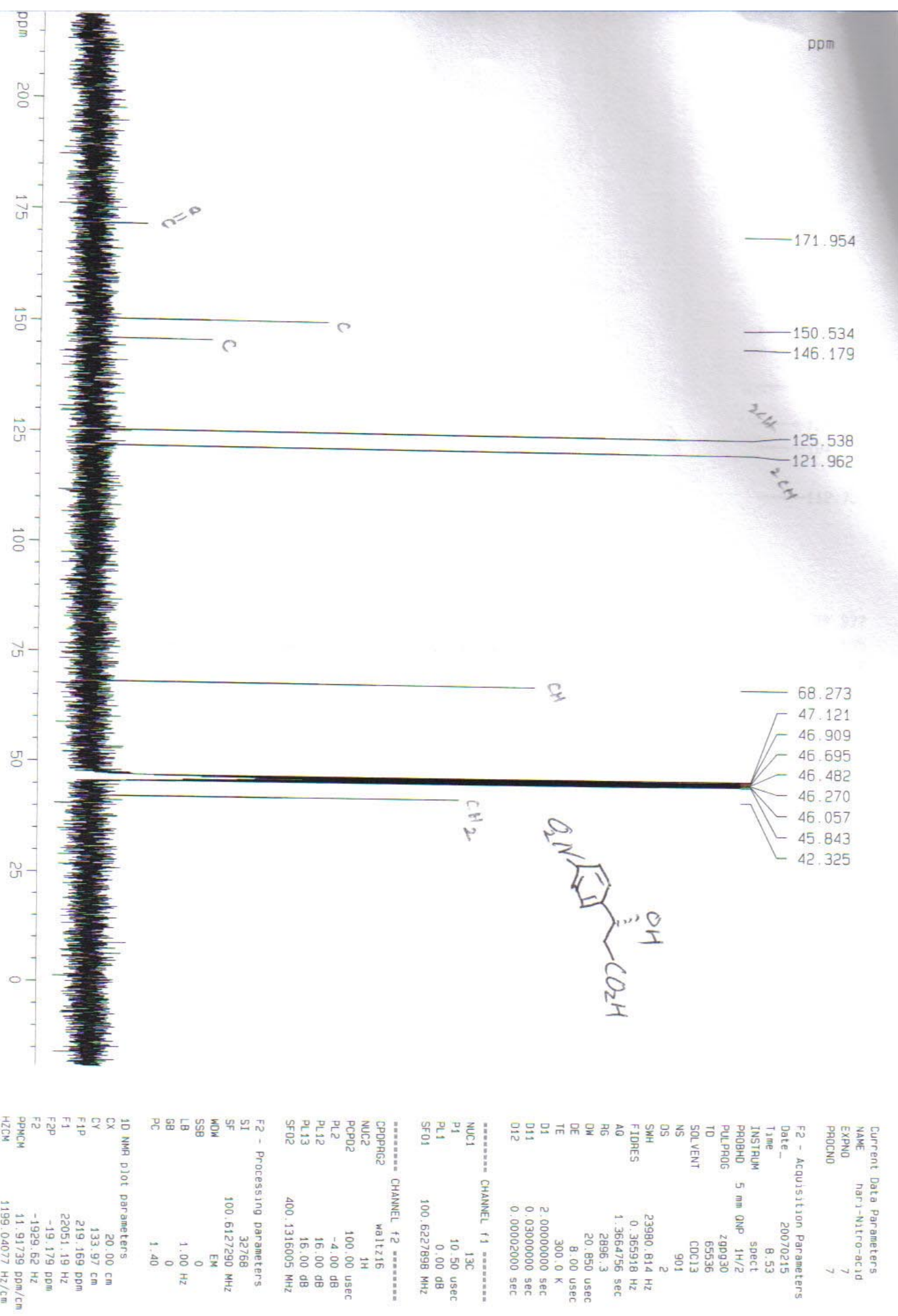




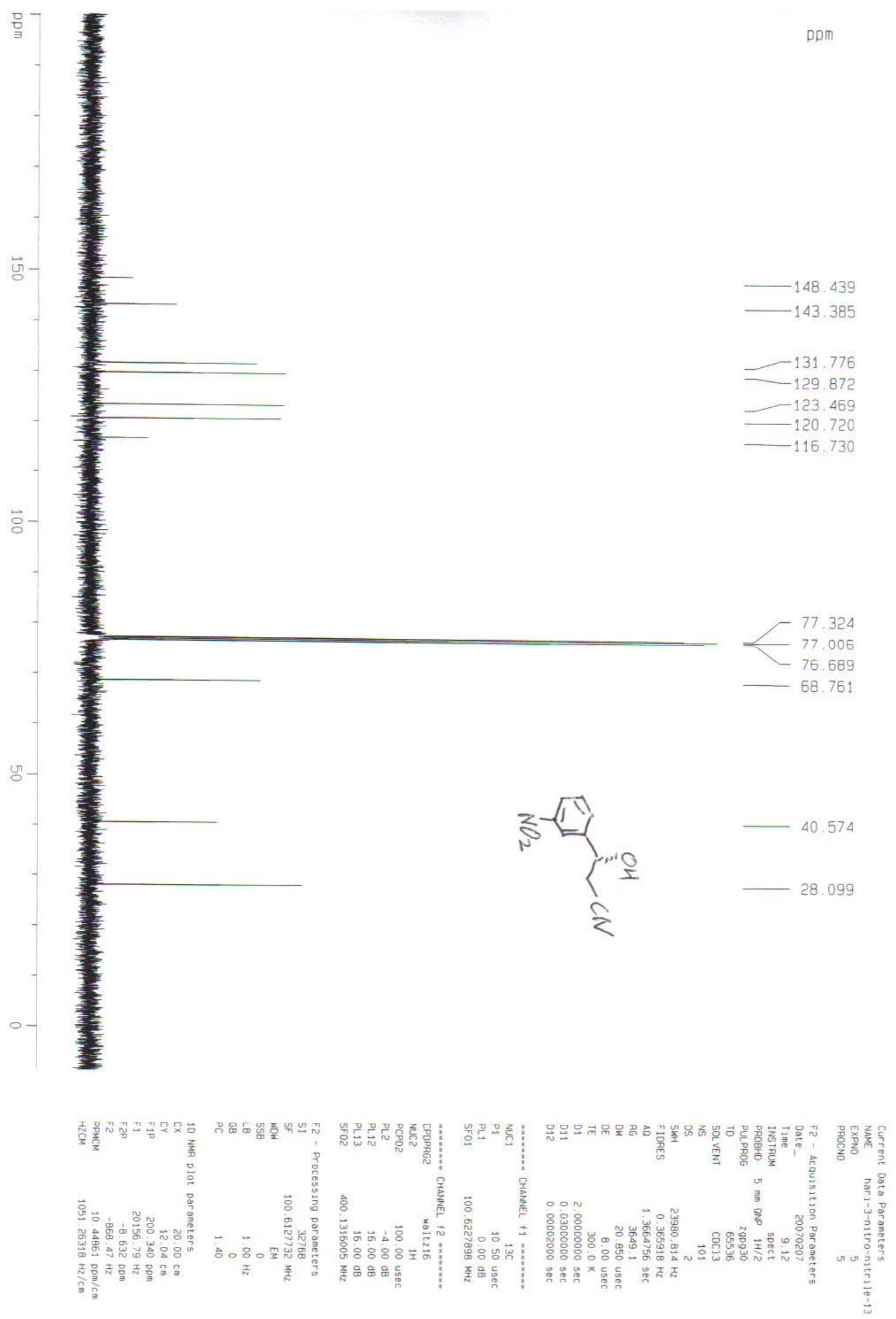




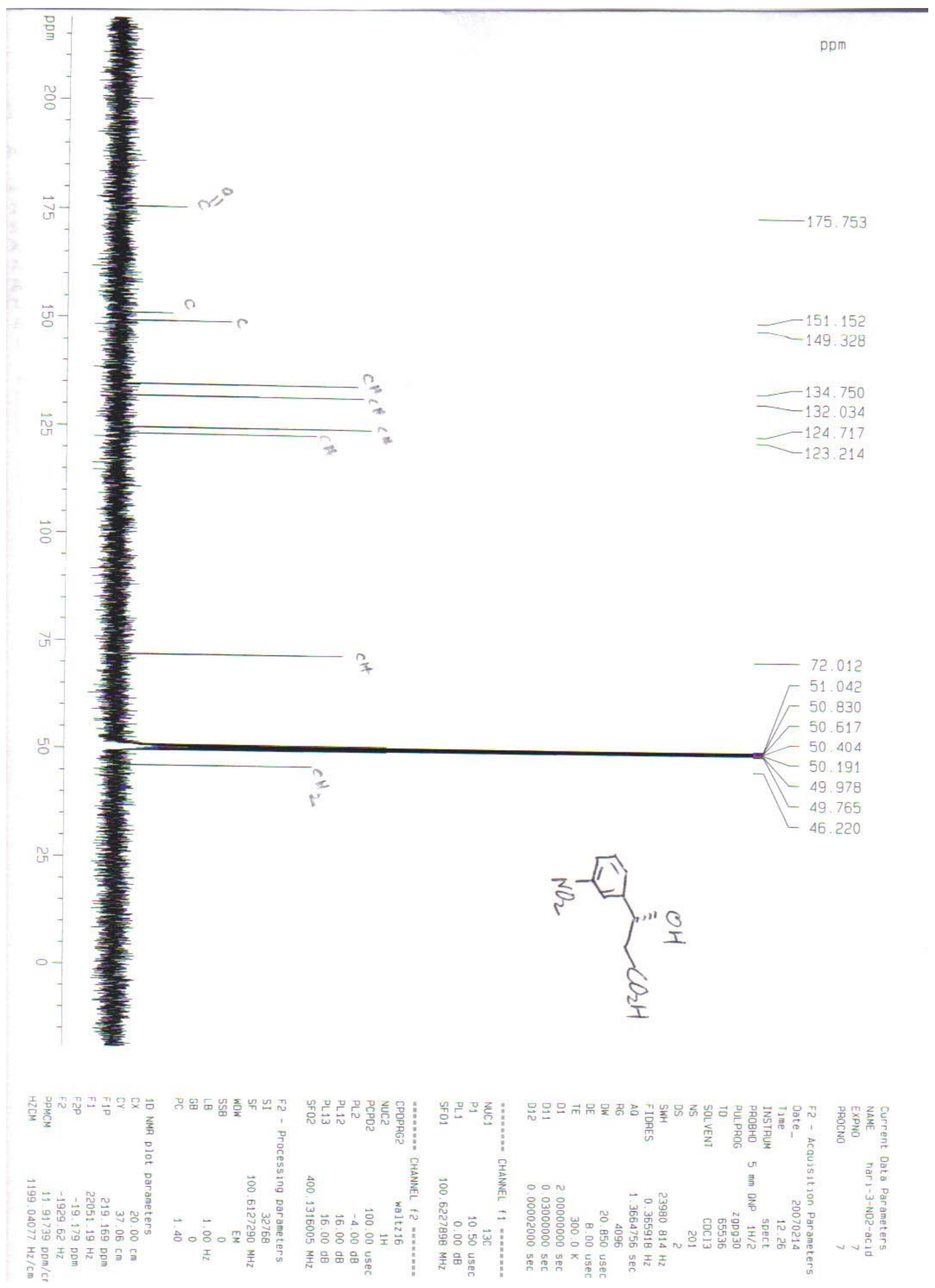




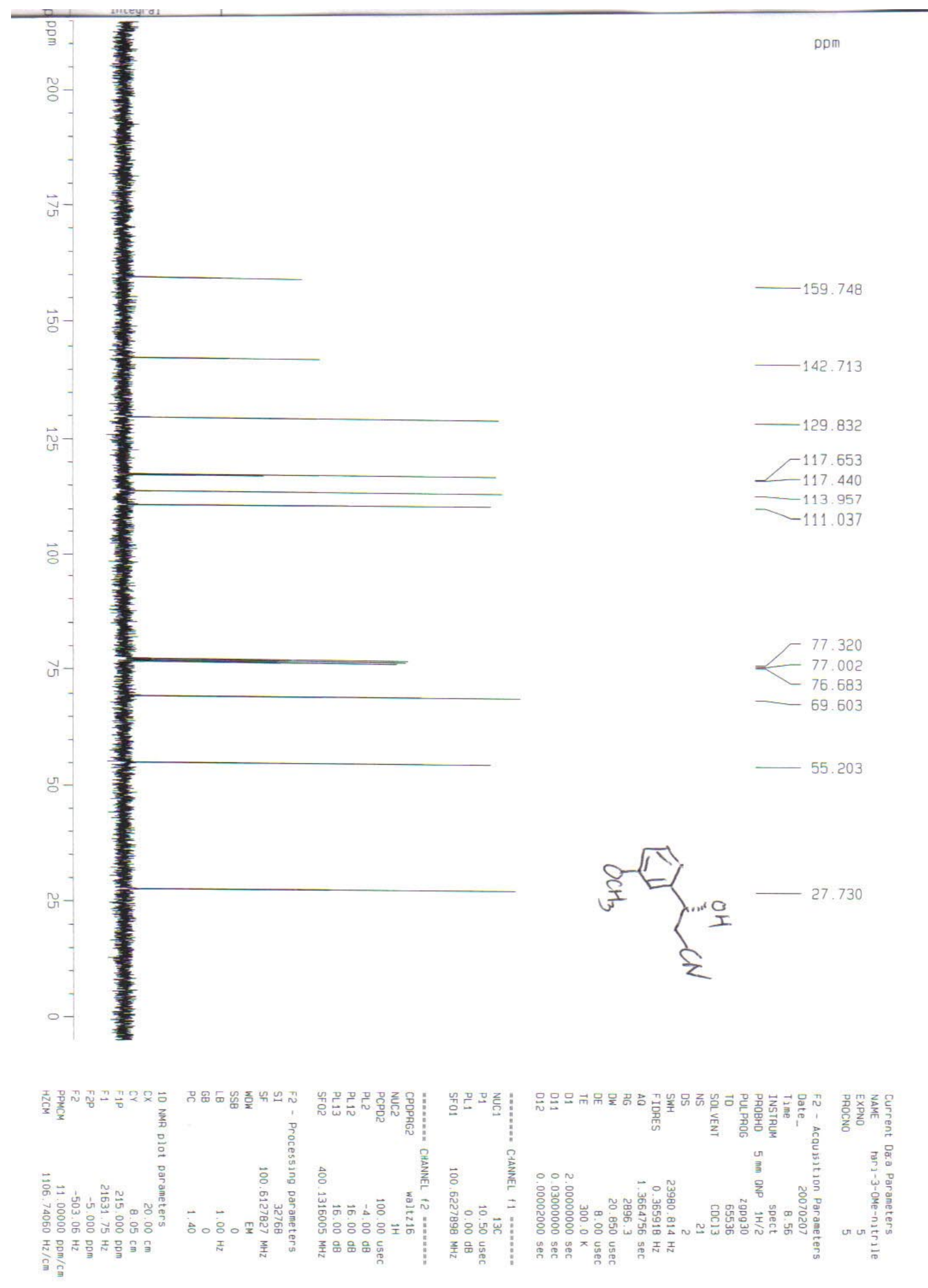




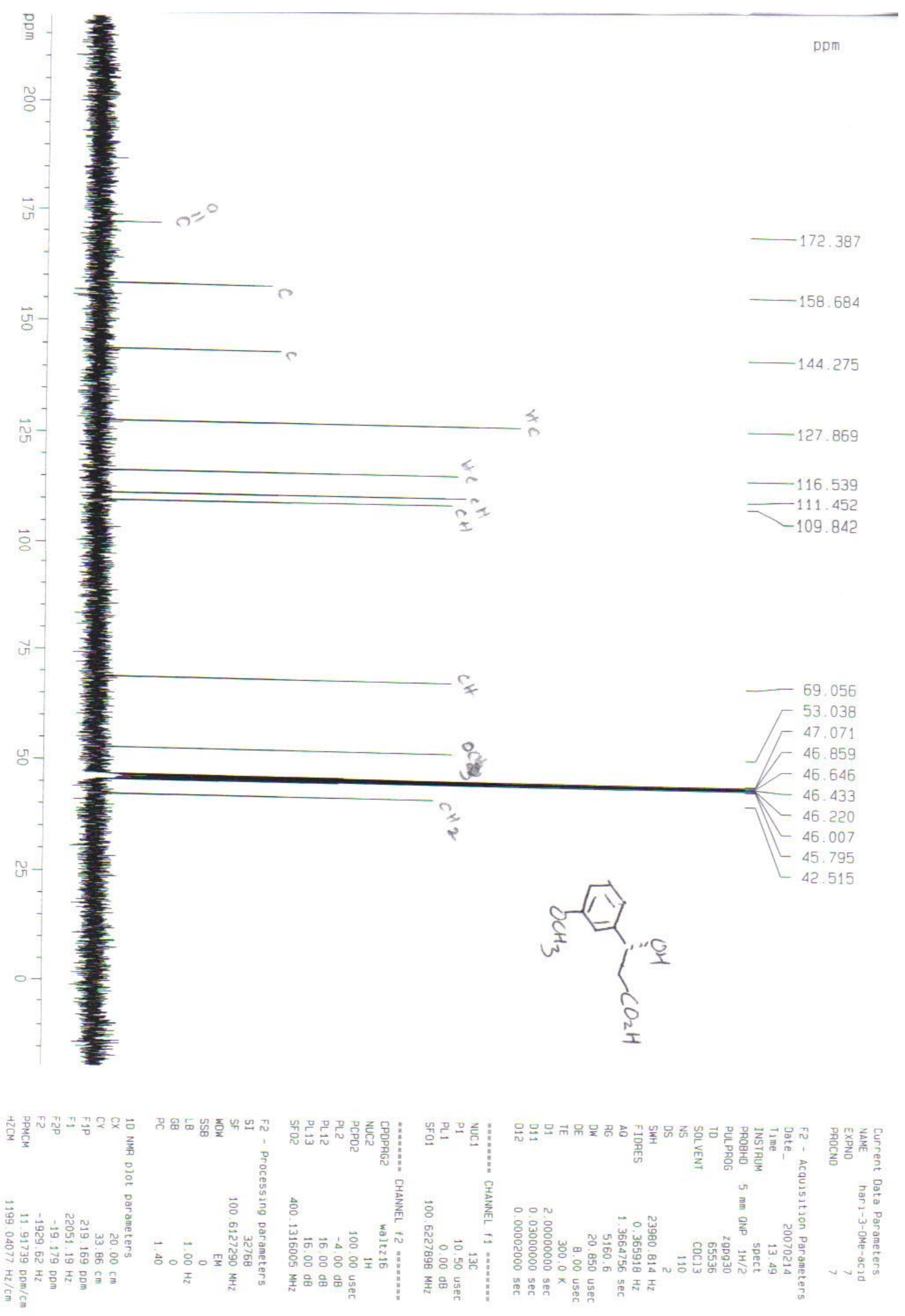


